\title{
Dyonic AdS black holes from magnetohydrodynamics
}

\author{
Marco M. Caldarelli, ${ }^{1,2}$ Óscar J. C. Dias ${ }^{1,3}$ and Dietmar Klemm ${ }^{4}$ \\ ${ }^{1}$ Departament de Física Fonamental, Universitat de Barcelona, \\ Marti i Franquès 1, E-08028 Barcelona \\ ${ }^{2}$ Instituut voor Theoretische Fysica, Katholieke Universiteit Leuven, \\ Celestijnenlaan 200D B-3001 Leuven, Belgium \\ ${ }^{3}$ Dept. de Física e Centro de Física do Porto, Faculdade de Ciências da Universidade do Porto, \\ Rua do Campo Alegre 687, 4169 - 007 Porto, Portugal \\ ${ }^{4}$ Dipartimento di Fisica dell'Università di Milano, \\ Via Celoria 16, I-20133 Milano and \\ INFN, Sezione di Milano, Via Celoria 16, I-20133 Milano. \\ marco.caldarelli@fys.kuleuven.be, oscar.dias@fc.up.pt, dietmar.klemm@mi.infn.it
}

\begin{abstract}
We use the AdS/CFT correspondence to argue that large dyonic black holes in anti-de Sitter spacetime are dual to stationary solutions of the equations of relativistic magnetohydrodynamics on the conformal boundary of AdS. The dyonic Kerr-Newman-AdS 4 solution corresponds to a charged diamagnetic fluid not subject to any net Lorentz force, due to orthogonal magnetic and electric fields compensating each other. The conserved charges, stress tensor and R-current of the fluid are shown to be in exact agreement with the corresponding quantities of the black hole. Furthermore, we obtain stationary solutions of the Navier-Stokes equations in four dimensions, which yield predictions for (yet to be constructed) charged rotating black strings in $\mathrm{AdS}_{5}$ carrying nonvanishing momentum along the string. Finally, we consider Scherk-Schwarz reduced AdS gravity on a circle. In this theory, large black holes and black strings are dual to lumps of deconfined plasma of the associated CFT. We analyze the effects that a magnetic field introduces in the Rayleigh-Plateau instability of a plasma tube, which is holographically dual to the Gregory-Laflamme instability of a magnetically charged black string.
\end{abstract}




\section{Contents}

1 Introduction $\quad 1$

2 Magnetohydrodynamics 3

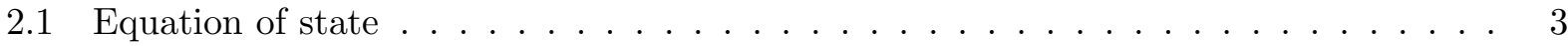

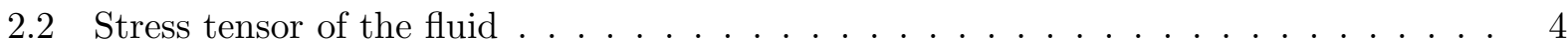

2.3 MHD equations from gravity $\ldots \ldots \ldots \ldots \ldots \ldots$

3 AdS black holes and black strings from MHD $\quad 9$

3.1 Static dyonic black holes in $\mathrm{AdS}_{4} \ldots \ldots \ldots \ldots \ldots \ldots$

3.2 Kerr-Newman-AdS $\mathrm{Ad}_{4} \ldots \ldots \ldots \ldots \ldots \ldots \ldots \ldots$

3.3 Black strings in $\mathrm{AdS}_{5} \ldots \ldots \ldots \ldots \ldots \ldots \ldots \ldots$

4 Gregory-Laflamme on magnetized plasma tubes $\quad 22$

4.1 Description of the problem. Scherk-Schwarz compactification of a CFT . . . . . . 22

4.2 MHD for Scherk-Schwarz plasmas . . . . . . . . . . . . . . . . 24

4.3 Equation of state for a Scherk-Schwarz plasma . . . . . . . . . . . . . . . 25

4.4 Rayleigh-Plateau instability in a magnetic background . . . . . . . . . . . . . . 26

4.5 Unstable plasma tubes and their gravitational duals . . . . . . . . . . . . . . 30

4.6 Regime of validity . . . . . . . . . . . . . . . . . . 33

\section{Introduction}

The AdS/CFT correspondence (cf. [1] for a review) has provided us with a powerful tool to get insight into the strong coupling dynamics of certain field theories by studying classical gravity. In the long wavelength limit, it is reasonable to expect that these interacting field theories admit an effective description in terms of hydrodynamics. One can thus conversely use the equations of fluid mechanics (which, in simple contexts, are much easier to solve than the full set of Einstein's equations) in order to make predictions for the gravity side. This was done in [2], where the fluid configurations dual to (yet to be discovered) black rings in (Scherk-Schwarz compactified) AdS were constructed ${ }^{1}$.

More recently, it was shown in [4] (cf. also [5] for the four-dimensional case) that the equations of hydrodynamics (i. e., the Navier-Stokes equations) can also be derived directly from Einstein's equations with negative cosmological constant, without making any use of string theory, from which these ideas originally emerged.

The correspondence between gravity in asymptotically AdS spaces and fluid mechanics has interesting consequences: For instance, it is well known that under certain conditions fluids are affected by the so-called Rayleigh-Plateau instability (responsible e. g. for the pinch-off of thin water jets from kitchen taps), and one might ask what the gravity dual of (the relativistic analogue

\footnotetext{
${ }^{1}$ For a discussion of plasma lumps dual to black saturns in AdS cf. [3].
} 
of) this instability is. This point was studied recently in [6], where it was argued that the gravity dual is the Gregory-Laflamme instability [7].

In this paper, we shall consider a more general setting in which the charged fluid moves in external magnetic fields. The fluid is thus described by the equations of magnetohydrodynamics (MHD). Such a generalization is of interest for several reasons: First, there are two possible fall-off conditions for a bulk U(1) gauge field: A normalizable mode that corresponds to a VEV of the dual operator (an R-current), and a non-normalizable one corresponding to the application of an external gauge field, that can be thought of as an electromagnetic field. $(2+1)$-dimensional field theories deformed in this way, that are dual to magnetically charged $\mathrm{AdS}_{4}$ black holes, have become fashionable recently in the context of a possible holographic realization of condensed matter phenomena like superconductivity [8,9], the Hall effect [10] and the Nernst effect [11]. In the long wavelength regime a quantum field theory in presence of external gauge fields is expected to be described by MHD. Second, solving the MHD equations (which, under certain symmetry assumptions, might be much easier than solving the full set of Einstein-Maxwell equations) can be helpful for constructing new magnetically charged black hole solutions in AdS. Furthermore, the phase structure of such AdS black holes can be studied in a simplified setting using magnetohydrodynamics.

We should stress that the magnetic field entering the MHD equations is nondynamical, so that there are no Maxwell equations on the boundary. Note however that, in the same way in which one can promote the CFT metric to a dynamical field [12], it should be possible to add dynamics also to the magnetic field, although we will not try to do this here.

The remainder of this paper is organized as follows: In section 2, we review general aspects of MHD, like the equation of state and the stress tensor of the fluid under the influence of external magnetic fields. Moreover, we show how the MHD equations emerge from Einstein-Maxwell-AdS gravity. In the following section, we generalize the results of [13] to the case of nonvanishing magnetic fields. We first consider static dyonic black holes in $\mathrm{AdS}_{4}$. In an appropriate limit (when the horizon radius $r_{h}$ is much larger than the AdS curvature radius $\ell$ ), these black holes are effectively described by magnetohydrodynamics. Conformal invariance and extensivity imply that the grandcanonical partition function has to take the form

$$
\frac{1}{V} \ln \mathcal{Z}_{g c}=T^{2} h\left(\zeta / T, B / T^{2}\right),
$$

where $\zeta$ denotes a chemical potential conjugate to the $\mathrm{U}(1)$ electric charge of the fluid, $B$ is the applied magnetic field, and $V$ and $T$ represent the volume and the overall temperature of the fluid respectively. We then show that, using the function $h$ that can be read off from the partition function of static black holes as an input into the MHD equations, we can exactly reproduce the conserved charges, boundary stress tensor and R-currents associated to the rotating dyonic KerrNewman-AdS solutions. Their thermodynamics is very simple: It is summarized by the partition function

$$
\frac{1}{V} \ln \mathcal{Z}_{g c}=\frac{T^{2} h\left(\zeta / T, B \Xi / T^{2}\right)}{\Xi},
$$

with $\Xi=1-\omega^{2} \ell^{2}$, and $\omega$ is the angular velocity of the black hole.

After that, we solve the Navier-Stokes equations on $\mathbb{R} \times S^{1} \times S^{2}, \mathbb{R} \times S^{1} \times \mathbb{R}^{2}$ or $\mathbb{R} \times S^{1} \times H^{2}$. This yields predictions for (yet to be constructed) charged rotating black strings in $\mathrm{AdS}_{5}$ with 
momentum along the string.

In section 4 we consider perturbations around plasma tube solutions of the MHD equations on $\mathbb{R} \times \mathbb{R}^{2} \times S^{1}$, that are dual to magnetic black strings on Scherk-Schwarz compactified AdS $_{6}$. These plasma tubes suffer from the long wavelength Rayleigh-Plateau instability, which is shown to be weakened by the presence of a magnetic background. This is exactly what one expects from the known studies of the Gregory-Laflamme instability for magnetically charged black strings in asymptotically flat space, to which the considered SS-compactified AdS black strings are similar.

Throughout this paper we use calligraphic letters $\mathcal{T}, \mathcal{V}, \mathcal{B}, \mathcal{S}, \ldots$ to indicate local thermodynamic quantities, whereas $T, V, B, S, \ldots$ refer to the whole fluid configuration. $\mu$ and $\zeta$ are local and global chemical potentials respectively.

Note added: While this paper was in preparation, ref. [14] appeared that partially overlaps with our section 2.3.

\section{Magnetohydrodynamics}

\subsection{Equation of state}

We would like to know how the equation of state $\mathcal{P}=\rho /(d-1)$ of a conformal fluid in $d$ dimensions changes in presence of a magnetic field $\mathcal{B}$. To this end, consider the grandcanonical potential

$$
\Phi=\mathcal{E}-\mathcal{T S}-\mu \mathcal{R}=\Phi(\mathcal{T}, \mathcal{V}, \mu, \mathcal{B})
$$

It follows from conformal invariance and extensivity that

$$
\Phi=-\mathcal{V} \mathcal{T}^{d} h(\nu, b)
$$

where we defined $\nu=\mu / \mathcal{T}$ and $b=\mathcal{B} / \mathcal{T}^{2}$. Note that $\mu$ and $\mathcal{B}$ have mass dimension one and two respectively, so that $\nu$ and $b$ are dimensionless. Equation (2.2) defines the function $h(\nu, b)$. From $(2.2)$ it is also clear that

$$
\Phi\left(\lambda \mathcal{T}, \lambda^{1-d} \mathcal{V}, \lambda \mu, \lambda^{2} \mathcal{B}\right)=\lambda \Phi(\mathcal{T}, \mathcal{V}, \mu, \mathcal{B})
$$

If we derive this with respect to $\lambda$, set $\lambda=1$ and use

$$
\frac{\partial \Phi}{\partial \mathcal{T}}=-\mathcal{S}, \quad \frac{\partial \Phi}{\partial \mathcal{V}}=-\mathcal{P}, \quad \frac{\partial \Phi}{\partial \mu}=-\mathcal{R}, \quad \frac{\partial \Phi}{\partial \mathcal{B}}=-\mathcal{V} \mathcal{M}
$$

where $\mathcal{M}$ is the magnetization density, we obtain

$$
-\mathcal{S} \mathcal{T}-(1-d) \mathcal{P} \mathcal{V}-\mathcal{R} \mu-2 \mathcal{V} \mathcal{M B}=\Phi
$$

Using the definition (2.1) of $\Phi$, this yields the equation of state

$$
\rho=(d-1) \mathcal{P}-2 \mathcal{B M} .
$$

We will see below that large dyonic black holes in $\mathrm{AdS}_{4}$ indeed have a grandcanonical potential of the form (2.2), with $d=3$. 


\subsection{Stress tensor of the fluid}

We want to determine now the general form of the stress tensor for our fluid, and we will do this in a derivative expansion in the fluid velocity $u^{\mu}$, following [15]. The zero order term corresponds to a perfect fluid (under the influence of external electromagnetic fields $F_{\mu \nu}^{I}$, labelled by the index $I$ ), with stress tensor, charge currents and entropy current given respectively by (cf. e. g. [11])

$$
T^{\mu \nu}=\rho u^{\mu} u^{\nu}+\mathcal{P} \Pi^{\mu \nu}-\mathcal{M}_{I}^{\mu \lambda} F_{\lambda}^{I \nu}, \quad J_{I}^{\mu}+\nabla_{\sigma} \mathcal{M}_{I}^{\sigma \mu}=r_{I} u^{\mu}, \quad J_{S}^{\mu}=s u^{\mu}
$$

where we have introduced the projection tensor

$$
\Pi^{\mu \nu}=g^{\mu \nu}+u^{\mu} u^{\nu}
$$

on the directions orthogonal to $u^{\mu} . \quad \rho=\rho\left(\mathcal{T}, \mu^{I}, \mathcal{B}^{J}\right)$ is the rest frame energy density, $r_{I}=$ $r_{I}\left(\mathcal{T}, \mu^{J}, \mathcal{B}^{K}\right)$ and $s=s\left(\mathcal{T}, \mu^{I}, \mathcal{B}^{J}\right)$ are the rest frame charge- and entropy densities, while $\mu^{I}$ and $\mathcal{B}^{J}$ denote the chemical potentials and magnetic fields respectively. The polarization tensor $\mathcal{M}_{I}^{\mu \lambda}$ is defined by

$$
\mathcal{M}_{I}^{\mu \lambda}=-\frac{1}{\mathcal{V}} \frac{\partial \Phi}{\partial F_{\mu \lambda}^{I}} .
$$

Note that the microscopic currents (polarization currents) are given by

$$
J_{I \text { micr. }}^{\mu}=-\nabla_{\sigma} \mathcal{M}_{I}^{\sigma \mu}
$$

whereas $J_{I}^{\mu}$ represents the total current density, so that the combination $J_{I}^{\mu}+\nabla_{\sigma} \mathcal{M}_{I}^{\sigma \mu}$ appearing in (2.7) is the macroscopic or transport current. Notice also that the extra contribution $-\mathcal{M}_{I}^{\mu \lambda} F^{I \nu}{ }_{\lambda}$ to the stress tensor in (2.7) comes from the coupling of the polarization to the electromagnetic field.

The first subleading order in the derivative expansion of the MHD equations describes dissipative phenomena like viscosity and diffusion. Lorentz invariance and the requirement that the entropy is non-decreasing determine the form of the stress tensor and the currents. Let us introduce diffusion currents $q_{I}^{\mu}$,

$$
J_{I}^{\mu}+\nabla_{\sigma} \mathcal{M}_{I}^{\sigma \mu}=r_{I} u^{\mu}+q_{I}^{\mu},
$$

with the constraint $u_{\mu} q_{I}^{\mu}=0$, meaning that the diffusive process is purely spatial according to an observer comoving with the fluid element. Next we introduce the heat flux $q^{\mu}$ and the viscous stress tensor, decomposed in a symmetric traceless part $\tau^{\mu \nu}$ and a trace $\tau$, such that

$$
T^{\mu \nu}=(\mathcal{P}+\tau) \Pi^{\mu \nu}+\rho u^{\mu} u^{\nu}-\mathcal{M}_{I}^{\mu \lambda} F_{\lambda}^{I \nu}+q^{\mu} u^{\nu}+q^{\nu} u^{\mu}+\tau^{\mu \nu} .
$$

Again these quantities are purely spatial, and verify the constraints

$$
u_{\mu} q^{\mu}=0, \quad u_{\mu} \tau^{\mu \nu}=0
$$

Note that the new fields $q_{I}^{\mu}, q^{\mu}, \tau$ and $\tau^{\mu \nu}$ are first order in $\nabla u$. Finally the entropy flux is a linear combination of all the available vectors that are at most first order in $\nabla u$. Therefore

$$
J_{S}^{\mu}=s u^{\mu}+\beta q^{\mu}-\lambda^{I} q_{I}^{\mu},
$$


where the scalars $\beta$ and $\lambda^{I}$ are functions of the thermodynamic parameters.

To determine the most general form allowed for the newly introduced quantities we impose the second law of thermodynamics. To have a non-decreasing total entropy the four-divergence of the entropy flux must be positive,

$$
\nabla_{\mu} J_{S}^{\mu} \geq 0
$$

Projecting the MHD equations on $u^{\mu}$ we obtain

$$
u_{\mu} \nabla_{\nu} T^{\mu \nu}=u^{\mu} J_{I}^{\nu} F_{\mu \nu}^{I},
$$

which can be cast, with some straightforward manipulations, in the form ${ }^{2}$

$$
\begin{aligned}
(\rho+\mathcal{P}) \vartheta= & -\tau \vartheta-u^{\mu} \nabla_{\mu} \rho+u_{\mu} u^{\nu} \nabla_{\nu} q^{\mu}-\nabla_{\mu} q^{\mu}+u_{\mu} \nabla_{\nu} \tau^{\mu \nu} \\
& -\frac{1}{2} u^{\mu} \mathcal{M}_{I}^{\nu \lambda} \nabla_{\mu} F_{\nu \lambda}^{I}-u^{\mu} F_{\mu \nu}^{I}\left(J_{I}^{\nu}+\nabla_{\sigma} \mathcal{M}_{I}^{\sigma \nu}\right)
\end{aligned}
$$

with the expansion $\vartheta=\nabla_{\mu} u^{\mu}$.

We have the Euler relation

$$
\rho+\mathcal{P}=s \mathcal{T}+\mu^{I} r_{I}
$$

which implies the Gibbs-Duhem relation

$$
\mathrm{d} \mathcal{P}=s \mathrm{~d} \mathcal{T}+r_{I} \mathrm{~d} \mu^{I}+\mathcal{M}_{I} \mathrm{~d} \mathcal{B}^{I}
$$

with the magnetization densities $\mathcal{M}_{I}$. This yields

$$
s \nabla_{\mu} \mathcal{T}=\nabla_{\mu} \mathcal{P}-r_{I} \nabla_{\mu} \mu^{I}-\mathcal{M}_{I} \nabla_{\mu} \mathcal{B}^{I}
$$

The entropy density is a function of $\rho, r_{I}$ and $\mathcal{B}^{J}$, so that its gradient reads

$$
\nabla_{\mu} s=\left(\frac{\partial s}{\partial \rho}\right)_{r_{I}, \mathcal{B}^{J}} \nabla_{\mu} \rho+\left(\frac{\partial s}{\partial r_{I}}\right)_{\rho, \mathcal{B}^{J}} \nabla_{\mu} r_{I}+\left(\frac{\partial s}{\partial \mathcal{B}^{J}}\right)_{r_{I}, \rho} \nabla_{\mu} \mathcal{B}^{J}=\frac{1}{\mathcal{T}} \nabla_{\mu} \rho-\frac{\mu^{I}}{\mathcal{T}} \nabla_{\mu} r_{I}+\frac{\mathcal{M}_{J}}{\mathcal{T}} \nabla_{\mu} \mathcal{B}^{J}
$$

Then

$$
\begin{aligned}
\nabla_{\mu} J_{S}^{\mu}= & \frac{1}{\mathcal{T}} u^{\mu} \nabla_{\mu} \rho-\frac{\mu^{I}}{\mathcal{T}} u^{\mu} \nabla_{\mu} r_{I}+\frac{\mathcal{M}_{J}}{\mathcal{T}} u^{\mu} \nabla_{\mu} \mathcal{B}^{J}+\left(\frac{\mathcal{P}+\rho-\mu^{I} r_{I}}{\mathcal{T}}\right) \vartheta \\
& +\beta \nabla_{\mu} q^{\mu}+q^{\mu} \nabla_{\mu} \beta-\lambda^{I} \nabla_{\mu} q_{I}^{\mu}-q_{I}^{\mu} \nabla_{\mu} \lambda^{I}
\end{aligned}
$$

The conservation of the charge currents $\nabla_{\mu} J_{I}^{\mu}=0$ yields the relation ${ }^{3}$

$$
u^{\mu} \nabla_{\mu} r_{I}=-\nabla_{\mu} q_{I}^{\mu}-r_{I} \vartheta
$$

\footnotetext{
${ }^{2}$ To get this, one has to use $\mathcal{M}_{I}^{\mu \lambda}=\chi_{I J} F^{J \mu} \lambda$, with the susceptibilities $\chi_{I J}=\chi_{J I}$, as well as the Bianchi identities for $F^{I}$.

${ }^{3}$ Note that the polarization current is separately conserved, $\nabla_{\mu} \nabla_{\sigma} \mathcal{M}^{\sigma \mu}=0$.
} 
that, together with (2.17), can be used to put the divergence of the entropy flux in the form

$$
\begin{aligned}
\nabla_{\mu} J_{S}^{\mu}= & q^{\mu}\left(\nabla_{\mu} \beta-\frac{1}{\mathcal{T}} u^{\nu} \nabla_{\nu} u_{\mu}\right)+\left(\beta-\frac{1}{\mathcal{T}}\right) \nabla_{\mu} q^{\mu}-\left(\lambda^{I}-\frac{\mu^{I}}{\mathcal{T}}\right) \nabla_{\mu} q_{I}^{\mu} \\
& -q_{I}^{\mu}\left(\nabla_{\mu} \lambda^{I}-\frac{1}{\mathcal{T}} F_{\mu \nu}^{I} u^{\nu}\right)-\frac{\tau}{\mathcal{T}} \vartheta-\frac{1}{\mathcal{T}} \tau^{\mu \nu} \nabla_{\nu} u_{\mu}
\end{aligned}
$$

Note that, in order to get $(2.24)$, we assumed that the grandcanonical potential $\Phi$ depends on $F^{I}$ only through the invariant $F_{\mu \nu}^{I} F^{I \mu \nu}$ (no summation over $I$ ), and we defined $\left(\mathcal{B}^{I}\right)^{2}$ to be given (up to a prefactor) by $F_{\mu \nu}^{I} F^{I \mu \nu}$. This leads to

$$
-\frac{1}{2} \mathcal{M}_{I}^{\nu \lambda} \nabla_{\mu} F_{\nu \lambda}^{I}=-\mathcal{M}_{I} \nabla_{\mu} \mathcal{B}^{I}
$$

We ensure that the right hand side of $(2.24)$ is positive or vanishing by requiring that each term be positive or vanishing. This can be easily achieved by choosing

$$
\beta=\frac{1}{\mathcal{T}}, \quad \lambda^{I}=\frac{\mu^{I}}{\mathcal{T}} .
$$

Then, the diffusion currents are given by

$$
q_{I}^{\mu}=-D_{I J} \Pi^{\mu \nu}\left[\nabla_{\nu}\left(\frac{\mu^{J}}{\mathcal{T}}\right)-\frac{1}{\mathcal{T}} F_{\nu \sigma}^{J} u^{\sigma}\right],
$$

where we have introduced the projector $\Pi^{\mu \nu}$ to ensure that the diffusion currents be spacelike, and the diffusion matrix $D_{I J}$ is positive definite. Moreover, we set

$$
\tau=-\zeta \nabla_{\mu} u^{\mu}
$$

where $\zeta \geq 0$ is the bulk viscosity, and the heat flux is given by

$$
q^{\mu}=-\kappa \mathcal{T} \Pi^{\mu \nu}\left(\frac{1}{\mathcal{T}} \nabla_{\nu} \mathcal{T}+u^{\sigma} \nabla_{\sigma} u_{\nu}\right)
$$

with $\kappa \geq 0$ the thermal conductivity of the fluid. Finally,

$$
\tau^{\mu \nu}=-\eta \sigma^{\mu \nu},
$$

with $\eta \geq 0$ the shear viscosity of the fluid and $\sigma^{\mu \nu}$ the shear tensor,

$$
\sigma^{\mu \nu}=\frac{1}{2}\left(\Pi^{\sigma \nu} \nabla_{\sigma} u^{\mu}+\Pi^{\sigma \mu} \nabla_{\sigma} u^{\nu}\right)-\frac{1}{3} \Pi^{\mu \nu} \vartheta .
$$

With these definitions, we find

$$
\nabla_{\mu} J_{S}^{\mu}=\frac{q^{\mu} q_{\mu}}{\kappa \mathcal{T}^{2}}+\frac{\zeta}{\mathcal{T}} \vartheta^{2}+\left(D^{-1}\right)^{I J} q_{I}^{\mu} q_{J \mu}+\frac{\tau^{\mu \nu} \tau_{\mu \nu}}{\eta \mathcal{T}} \geq 0
$$

which is positive by construction. Notice finally that the stress tensor in (2.7) is traceless on account of the equation of state (2.6). 


\subsection{MHD equations from gravity}

In this section, we consider Einstein-Maxwell-AdS gravity in $D$ dimensions and show that at leading order the boundary theory dual to a charged $D$-dimensional AdS black hole reduces to magnetohydrodynamics in $d=D-1$ dimensions in the long wavelength sector. We follow the usual procedure to foliate asymptotically the spacetime with timelike hypersurfaces $\Sigma_{r}$ and regularize the action by adding appropriate boundary counterterms. Then, in the $r \rightarrow \infty$ limit, the Brown-York stress tensor has a finite limit, the renormalized holographic stress tensor of the dual CFT. The projection of the Einstein-Maxwell equations on $\Sigma_{r}$ then shows that this stress tensor together with the boundary gauge fields satisfy the equations of magnetohydrodynamics on the boundary. We can interpret this as the leading contribution in the derivative expansion to the boundary MHD equations describing the long wavelength sector of gravity.

The Einstein-Maxwell action with negative cosmological constant $\Lambda=-(D-2)(D-1) / 2 \ell^{2}$ reads

$$
I=\frac{1}{16 \pi G} \int d^{D} x \sqrt{-g}\left(R-2 \Lambda-\mathcal{F}_{M N} \mathcal{F}^{M N}\right)+I_{C S}+\frac{1}{8 \pi G} \int_{\Sigma_{R}} d^{d} x \sqrt{-h} K+I_{c t},
$$

where $I_{C S}$ is the Chern-Simons term, present in the $D=5$ case. $M, N, \ldots$ are bulk indices, whereas $\mu, \nu, \ldots$ refer to the boundary. In this subsection (and only here), we refer to the bulk gauge field as $\mathcal{F}_{M N}$ to distinguish it from the boundary gauge field. In all other sections of the paper we do not need such a distinction. $\Sigma_{R}$ denotes the boundary hypersurface $r=R$ with outward pointing unit normal $n^{M}$, and induced metric

$$
h_{M N}=g_{M N}-n_{M} n_{N} .
$$

$K$ is the trace of the extrinsic curvature defined by (with $\mathcal{D}_{M}$ the bulk covariant derivative)

$$
K_{M N}=h_{M}^{P} \mathcal{D}_{P} n_{N}
$$

Finally, $I_{c t}$ are the usual boundary counterterms needed to obtain a finite action in the limit $R \rightarrow \infty$. We shall not need the precise form of $I_{c t}$, but only the fact that their variation with respect to the metric is divergence-free,

$$
\hat{\nabla}^{\mu} \frac{\delta I_{c t}}{\delta h^{\mu \nu}}=0
$$

Here we have defined $\hat{\nabla}_{\mu}$ as the induced covariant derivative on $\Sigma_{R}$. Also, notice that additional terms can be added to $I_{c t}$ to handle the logarithmic divergences appearing for odd $D$, corresponding to the Weyl anomaly of the dual CFT. We can use the Fefferman-Graham expansion to write the metric of any asymptotically AdS spacetime near spatial infinity in the form

$$
d s^{2}=\frac{\ell^{2}}{r^{2}} d r^{2}+r^{2} g_{\mu \nu}(r, x) d x^{\mu} d x^{\nu}
$$

where

$$
g_{\mu \nu}=g_{\mu \nu}^{(0)}+\frac{1}{r^{2}} g_{\mu \nu}^{(2)}+\cdots+\frac{1}{r^{d}} g_{\mu \nu}^{(d)}+h_{\mu \nu}^{(d)} \frac{\ln r}{r^{d}}+\mathcal{O}\left(\frac{1}{r^{d+1}}\right)
$$


The coefficients $g_{\mu \nu}^{(a)}$ and $h_{\mu \nu}^{(d)}$ depend only on the boundary coordinates $x^{\mu}$ and the coefficient $h_{\mu \nu}^{(d)}$, related to the holographic Weyl anomaly, is present for odd $D$ only. The normal vector to the constant $r$ hypersurfaces is $n^{A} \partial_{A}=r / \ell \partial_{r}$ and the induced metric is $h_{\mu \nu}=\left.r^{2} g_{\mu \nu}\right|_{r=R}$. Then, the conformal boundary metric of $\mathrm{AdS}_{D}$ is obtained by taking the limit

$$
\gamma_{\mu \nu}=\lim _{R \rightarrow \infty} \frac{\ell^{2}}{R^{2}} h_{\mu \nu}
$$

Let us decompose the gauge field in the orthogonal component $\hat{J}^{M}$ and its projection $\hat{F}_{M N}$ on $\Sigma_{R}$,

$$
\mathcal{F}_{M N}=\hat{F}_{M N}+\frac{1}{2}\left(\hat{J}_{M} n_{N}-\hat{J}_{N} n_{M}\right),
$$

where

$$
\hat{J}^{M}=2 h^{M N} n^{P} \mathcal{F}_{N P}, \quad \hat{F}_{M N}=h_{M}^{P} h_{N}{ }^{Q} \mathcal{F}_{P Q} .
$$

The analysis of the asymptotic behavior of vector gauge fields $[16,17]$ shows that $\hat{F}_{\mu \nu}$ has a finite $R \rightarrow \infty$ limit, while the current $\hat{J}^{M}$ goes to zero like $r^{-d}$. Therefore, the renormalized background gauge field in the dual field theory, and the $R$-symmetry current read respectively

$$
F_{\mu \nu}=\lim _{R \rightarrow \infty} \hat{F}_{\mu \nu}, \quad J^{\mu}=\lim _{R \rightarrow \infty} \frac{\sqrt{-h}}{\sqrt{-\gamma}} \hat{J}^{\mu} .
$$

Now, by varying $I$ with respect to the bulk metric and gauge field, we obtain the equations of motion $^{4}$

$$
E_{M N}=G_{M N}+\Lambda g_{M N}-\mathcal{T}_{M N}=0, \quad \mathcal{D}^{M} \mathcal{F}_{M N}=0,
$$

where the stress tensor of the gauge field is given by

$$
\mathcal{T}_{M N}=2 \mathcal{F}_{M}{ }^{P} \mathcal{F}_{P N}-\frac{1}{2} \mathcal{F}^{2} g_{M N}
$$

Then, from the projection

$$
E_{M N} n^{M} h^{N}=0
$$

of the Einstein equations, we obtain, using Gauss-Codazzi

$$
\hat{\nabla}_{M}\left(K^{M}{ }_{N}-\delta^{M}{ }_{N} K\right)=\hat{F}_{N M} \hat{J}^{M} .
$$

We recognize in the term in parenthesis the Brown-York boundary stress tensor, which diverges as we take the $R \rightarrow \infty$ limit, but will give a finite limit - the holographic stress tensor $T^{\mu \nu}$ - once we take into account the counterterm contribution [18],

$$
\sqrt{-\gamma} \gamma^{\mu \nu} T_{\nu \rho}=\lim _{R \rightarrow \infty} \sqrt{-h} h^{\mu \nu}\left(K_{\nu \rho}-h_{\nu \rho} K+\frac{\delta I_{c t}}{\delta h^{\nu \rho}}\right)
$$

Since the counterterm contribution is divergence-free by (2.36), we can add its divergence to the left hand side of (2.46) and, after multiplying the equation by $\sqrt{-h}$, obtain a finite $R \rightarrow \infty$ limit that reads

$$
\nabla_{\mu} T_{\nu}^{\mu}=F_{\nu \mu} J^{\mu}
$$

\footnotetext{
${ }^{4}$ In $D=5$ one has to take into account that there is a Chern-Simons term and the Maxwell equations read $\mathrm{d}^{\star} \mathcal{F}+\frac{2}{\sqrt{3}} \mathcal{F} \wedge \mathcal{F}=0$.
} 
where $\nabla_{\mu}$ is the covariant derivative induced on the conformal boundary with metric $\gamma_{\mu \nu}$. On the other hand, using the definition (2.41) it follows that $\hat{\nabla}_{\mu} \hat{J}^{\mu}=0$, that becomes, in the $R \rightarrow \infty$ limit, the conservation law for the $R$-current,

$$
\nabla_{\mu} J^{\mu}=0
$$

Note that equations (2.48) and (2.49) are simply the Ward identities associated to the bulk diffeomorphism and gauge invariance respectively [19].

These conservation equations for the boundary stress-energy tensor and $R$-current become the equations for magnetohydrodynamics in the background field $F_{\mu \nu}$ when a large black hole is present in the bulk. Indeed, as noted in [20], stationary Kerr-AdS black holes have a holographic stress tensor that assumes the perfect fluid form. We will show in the next section that this is still the case for dyonic Kerr-Newman-AdS 4 black holes, and we believe it to be true in any dimension. This dual stress tensor, of the perfect fluid form, yields, when combined with equations (2.48) and (2.49), the equations of MHD. Then, if one perturbs these stationary solutions with long wavelength disturbances, in the spirit of [4], the horizon can still be decomposed into patches that tubewise extend to the boundary which are approximated by boosted pieces of black branes. Therefore, to leading order in the derivative expansion, large magnetically charged black holes are dual to a magnetohydrodynamic theory. We shall check this explicitely in the next section for dyonic $\mathrm{AdS}_{4}$ black holes. Higher orders in the perturbation theory produce higher-derivative dissipative terms in the stress tensor. A detailed analysis of this long wavelength sector of gravity, with a complete proof of the duality with MHD and the computation of the dual stress tensor up to third order in the derivative expansion has been performed by Hansen and Kraus in the $\mathrm{AdS}_{4}$ case and appeared during the last stages of preparation of this manuscript [14].

\section{AdS black holes and black strings from MHD}

\subsection{Static dyonic black holes in $\mathrm{AdS}_{4}$}

The equations of motion following from the Einstein-Maxwell action with negative cosmological constant $\Lambda=-3 \ell^{-2}$,

$$
I=\frac{1}{16 \pi G} \int d^{4} x \sqrt{-g}\left[R-F_{M N} F^{M N}-2 \Lambda\right],
$$

admit the static dyonic black hole solutions

$$
d s^{2}=-V(r) d t^{2}+\frac{d r^{2}}{V(r)}+r^{2}\left(d \theta^{2}+S(\theta)^{2} d \phi^{2}\right),
$$

where

$$
V(r)=\frac{r^{2}}{\ell^{2}}+k-\frac{2 m}{r}+\frac{q_{e}^{2}+q_{m}^{2}}{r^{2}}
$$

and

$$
S(\theta)=\left\{\begin{aligned}
\sin \theta, & k=1 \\
1, & k=0 \\
\sinh \theta, & k=-1
\end{aligned}\right.
$$


The horizon is thus $S^{2}(k=1), \mathbb{R}^{2}(k=0)$ or $H^{2}(k=-1) . m, q_{e}$ and $q_{m}$ denote the mass parameter, electric and magnetic charge respectively. The one-form gauge potential reads

$$
A_{t}=-\frac{q_{e}}{r}, \quad A_{\phi}=q_{m} \int S(\theta) d \theta .
$$

The strength of the magnetic field $B$ in the dual CFT can be obtained (up to rescaling by powers of $\ell$ ) by taking $r \rightarrow \infty$ in the expression for the bulk field strength. This leads to $B=q_{m} / \ell^{3}$. The electric charge density $\rho_{e}$ of the state in the field theory is given by $\rho_{e}=\left\langle J^{t}\right\rangle$, where $J^{\mu}$ is the $\mathrm{R}$-current that can be computed as follows. On-shell we have for the variation of the action with respect to the gauge potential

$$
\frac{\delta I}{\delta A_{N}} \delta A_{N}=-\frac{1}{4 \pi G} \int d^{4} x \partial_{M}\left(\sqrt{-g} F^{M N} \delta A_{N}\right)=-\frac{1}{4 \pi G} \int d^{3} x \sqrt{-h} n_{M} F^{M N} \delta A_{N},
$$

where $h$ denotes the induced metric on the boundary, and $n$ is the outward pointing unit normal to the boundary. One has thus in the limit of large $r$

$$
\frac{\delta I}{\delta A_{t}}=\frac{1}{4 \pi G} \frac{\ell q_{e}}{r^{3}}
$$

To get the CFT R-current, one has to rescale this by $r^{3} / \ell^{2}$, so that

$$
\left\langle J^{t}\right\rangle=\frac{\sqrt{2} N^{3 / 2} q_{e}}{6 \pi \ell^{3}},
$$

where we used the AdS/CFT dictionary

$$
\frac{1}{16 \pi G}=\frac{\sqrt{2} N^{3 / 2}}{24 \pi \ell^{2}}
$$

Note that the result (3.8) was obtained for $k=0$ in [10]. In order for the potential to be regular at the horizon $r=r_{h}, A_{t}$ must vanish there. This requires that we add the pure gauge term $\left(q_{e} / r_{h} \ell\right) d t$ to $A / \ell$. This term is non-normalizable and has the dual interpretation of adding a chemical potential for the electric charge, $\zeta=q_{e} / r_{h} \ell$, to the field theory [10]. The R-charge is $R=\rho_{e} V$, with the spatial volume ${ }^{5}$

$$
V=\ell^{2} \int d \theta d \phi S(\theta)
$$

This yields

$$
R=\frac{\sqrt{2} N^{3 / 2} q_{e} V}{6 \pi \ell^{3}}
$$

The entropy $S$ and energy $E$ of the black hole read

$$
S=\frac{A_{h}}{4 G}=\frac{\sqrt{2} N^{3 / 2} r_{h}^{2} V}{6 \ell^{4}},
$$

\footnotetext{
${ }^{5}$ In order to get a finite volume in the cases $k=0$ and $k=-1$, one has to compactify the horizon to a torus or a higher genus Riemann surface respectively.
} 


$$
E=\frac{m}{4 \pi G} \frac{V}{\ell^{2}}=\frac{\sqrt{2} N^{3 / 2} V}{12 \pi \ell^{4}}\left[\frac{r_{h}^{3}}{\ell^{2}}+k r_{h}+\frac{q_{e}^{2}+q_{m}^{2}}{r_{h}}\right] .
$$

In what follows, we shall be interested in a magnetohydrodynamical description of the above black holes. Like in [13], we may estimate the mean free path for the fluid as $l_{\operatorname{mfp}} \sim \eta / \rho$, where $\eta$ is the shear viscosity and $\rho$ is the energy density of the fluid. For fluids described by a gravitational dual, one has $\eta=s / 4 \pi$, where $s$ is the entropy density [21]. Consequently, $l_{\text {mfp }} \sim s / 4 \pi \rho$, and an MHD description will be valid if this value is much smaller than the radius of the $S^{2}$ or the $H^{2}$ curvature radius $^{6}, s / 4 \pi \rho \ll \ell$. Using the expressions for $S$ and $E$, this implies

$$
\frac{r_{h}}{\ell}+k \frac{\ell}{r_{h}}+\frac{\left(q_{e}^{2}+q_{m}^{2}\right) \ell}{r_{h}^{3}} \gg 1
$$

A sufficient condition for (3.14) to hold is $r_{h} / \ell \gg 1$, i. e. , for large black holes. In this case we can neglect the $k$-contribution to the energy, and the thermodynamic fundamental relation becomes

$$
E(S, V, R, B)=\frac{\sqrt{2} N^{3 / 2} V}{12 \pi}\left(\frac{6 S}{\sqrt{2} N^{3 / 2} V}\right)^{3 / 2}\left[1+\left(\frac{\pi R}{S}\right)^{2}+\frac{B^{2} N^{3} V^{2}}{18 S^{2}}\right] .
$$

One easily checks that

$$
\frac{\partial E}{\partial S}=T, \quad \frac{\partial E}{\partial R}=\zeta
$$

where

$$
T=\frac{V^{\prime}\left(r_{h}\right)}{4 \pi}=\frac{1}{4 \pi}\left[\frac{3 r_{h}}{\ell^{2}}-\frac{q_{e}^{2}+q_{m}^{2}}{r_{h}^{3}}\right]
$$

is the Hawking temperature of the black hole. From (3.15), we get the grandcanonical potential

$$
\Phi(T, V, \zeta, B)=E-T S-\zeta R=-V T^{3} h\left(\zeta / T, B / T^{2}\right),
$$

with the function $h$ given by

$$
h=\frac{\sqrt{2} N^{3 / 2}}{24 \pi}\left[H^{3}+H\left(\frac{\zeta}{T}\right)^{2}-\frac{3}{H}\left(\frac{B}{T^{2}}\right)^{2}\right],
$$

where $H\left(\zeta / T, B / T^{2}\right)=r_{h} /\left(\ell^{2} T\right)$ is determined by the fourth order equation

$$
3 H^{4}-4 \pi H^{3}-(\zeta / T)^{2} H^{2}-\left(B / T^{2}\right)^{2}=0,
$$

that follows from (3.17). Note that $\Phi$ has indeed the form (2.2), as it must be.

We now want to obtain the dyonic $\mathrm{AdS}_{4}$ black holes from MHD on $\mathbb{R} \times S^{2}, \mathbb{R} \times \mathbb{R}^{2}$ or $\mathbb{R} \times H^{2}$. The metric on the conformal boundary is given by

$$
d s^{2}=-d t^{2}+\ell^{2}\left(d \theta^{2}+S(\theta)^{2} d \phi^{2}\right),
$$

so that the only nonzero Christoffel symbols are

$$
\Gamma_{\phi \phi}^{\theta}=-S(\theta) S^{\prime}(\theta), \quad \Gamma_{\theta \phi}^{\phi}=\Gamma_{\phi \theta}^{\phi}=\frac{S^{\prime}(\theta)}{S(\theta)} .
$$

\footnotetext{
${ }^{6}$ In the (compactified) $k=0$ case, $l_{\text {mfp }}$ must be much smaller than the length of any torus cycle.
} 
For stationary, translationally invariant and axisymmetric configurations one has $\partial_{t} T^{\mu \nu}=\partial_{\phi} T^{\mu \nu}=$ 0 (we also assume $\partial_{t} F^{\mu \nu}=\partial_{\phi} F^{\mu \nu}=0$ ), and the MHD equations $\nabla_{\mu} T^{\mu \nu}=F_{\mu}^{\nu} J^{\mu}$ become thus

$$
\begin{aligned}
& \partial_{\theta} T^{\theta t}+\frac{S^{\prime}}{S} T^{\theta t}=F_{\mu}^{t} J^{\mu} \\
& \partial_{\theta} T^{\theta \theta}+\frac{S^{\prime}}{S} T^{\theta \theta}-S S^{\prime} T^{\phi \phi}=F^{\theta}{ }_{\mu} J^{\mu}, \\
& \partial_{\theta} T^{\theta \phi}+\frac{3 S^{\prime}}{S} T^{\theta \phi}=F^{\phi}{ }_{\mu} J^{\mu} .
\end{aligned}
$$

The macroscopic electric charge current and the entropy current are given respectively by

$$
J_{\text {macr. }}^{\mu}=J^{\mu}+\nabla_{\sigma} \mathcal{M}^{\sigma \mu}=r u^{\mu}, \quad J_{S}^{\mu}=s u^{\mu},
$$

where $u^{\mu}=\gamma(1, \vec{v})$ is the 3 -velocity of the fluid, $r$ denotes the electric charge density and $s$ is the rest frame entropy density. Both currents are conserved,

$$
\nabla_{\mu} J_{\text {macr. }}^{\mu}=\nabla_{\mu} J_{S}^{\mu}=0 .
$$

As there are no dissipative terms in the charge- and entropy currents, we have for the entropy

$$
S=\int d^{2} x \sqrt{-g} J_{S}^{t}=\int d \theta d \phi \ell^{2} S(\theta) s \gamma
$$

and for the electric charge

$$
R=\int d^{2} x \sqrt{-g} J_{\text {macr. }}^{t}=\int d \theta d \phi \ell^{2} S(\theta) r \gamma
$$

The Killing vectors of interest are $\partial_{t}$ (energy $E$ ) and $\partial_{\phi}$ (angular momentum $J$ on the $S^{2}, \mathbb{R}^{2}$ or $H^{2}$ ). The conserved charge related to a Killing vector $k$ is proportional to $\int d^{2} x \sqrt{-g} T^{t}{ }_{\mu} k^{\mu}$, and hence

$$
\begin{aligned}
E & =\int d \theta d \phi \ell^{2} S(\theta) T^{t t}, \\
J & =\int d \theta d \phi \ell^{4} S^{3}(\theta) T^{t \phi} .
\end{aligned}
$$

The shear tensor $\sigma^{\mu \nu}$, heat flux $q^{\mu}$ and diffusion current $q_{D}^{\mu}$ must vanish on any stationary solution of fluid dynamics. The requirement $\sigma^{\mu \nu}=0$ means that the fluid motion should be just a rigid rotation. By an $\mathrm{SO}(3)$ transformation ${ }^{7}$ we can can choose this rotation such that the 3 -velocity of the fluid is $u^{\mu}=\left(u^{t}, u^{\theta}, u^{\phi}\right)=\gamma(1,0, \omega)$ for some constant $\omega$. From $u^{\mu} u_{\mu}=-1$ and $\gamma=\left(1-v^{2}\right)^{-1 / 2}$ one obtains then $v^{2}=\omega^{2} \ell^{2} S^{2}(\theta)$.

The equilibrium fluid flow is symmetric under translations of $t$ and $\phi$, so that all thermodynamic quantities depend only on $\theta$. We now evaluate the expansion, acceleration, shear tensor, heat flux (2.29) and diffusion current (2.27), with the result

$$
\vartheta=0, \quad a^{\mu}=\left(0,-S(\theta) S^{\prime}(\theta) \gamma^{2} \omega^{2}, 0\right), \quad \sigma^{\mu \nu}=0,
$$

\footnotetext{
${ }^{7}$ Strictly speaking, an applied electromagnetic field breaks $\mathrm{SO}(3)$ invariance, so that our choice of $u^{\mu}$ implies consistency conditions on $F_{\mu \nu}$ (cf. (3.35) below).
} 


$$
\begin{gathered}
q^{\mu}=-\kappa \ell^{-2} \gamma\left(0, \frac{d}{d \theta}\left(\frac{\mathcal{T}}{\gamma}\right), 0\right) \\
q_{D}^{\mu}=-D \ell^{-2}\left(-\frac{1}{\mathcal{T}} F_{t \phi} \gamma \omega, \frac{d}{d \theta}\left(\frac{\mu}{\mathcal{T}}\right)-\frac{1}{\mathcal{T}} F_{\theta \sigma} u^{\sigma}, \frac{1}{\mathcal{T}} F_{t \phi} \gamma\right),
\end{gathered}
$$

where $\kappa$ and $D$ denote the thermal conductivity and the diffusion coefficient respectively, $\mathcal{T}$ is the local temperature and $\mu$ the local chemical potential. The requirement that $q^{\mu}$ and $q_{D}^{\mu}$ vanish implies that

$$
\mathcal{T}=\tau \gamma, \quad F_{t \phi}=0, \quad \frac{d}{d \theta}\left(\frac{\mu}{\gamma}\right)-\frac{1}{\gamma} F_{\theta \sigma} u^{\sigma}=0
$$

with $\tau$ constant. If $F_{\theta \sigma} u^{\sigma}=0$, the last equ. is solved by $\mu=\mathcal{T} \nu$, where $\nu$ is constant. The conditions (3.35) determine all the thermodynamic quantities as a function of the coordinate $\theta$. We now want to shew that this configuration solves the equations of magnetohydrodynamics. To this end, we first notice that the dissipative part of the energy-momentum tensor vanishes once (3.35) is imposed, so that all nonzero contributions to the stress tensor result from the perfect fluid part plus interaction with the external electromagnetic field, and read

$$
T^{\mu \nu}=\gamma^{2}\left(\begin{array}{ccc}
\rho+v^{2} \mathcal{P} & 0 & (\rho+\mathcal{P}) \omega \\
0 & \gamma^{-2} \ell^{-2} \mathcal{P} & 0 \\
(\rho+\mathcal{P}) \omega & 0 & \rho \omega^{2}+\mathcal{P} \ell^{-2} S^{-2}(\theta)
\end{array}\right)+T_{\text {int. }}^{\mu \nu},
$$

where $\mathcal{P}$ denotes the local pressure and $T_{\text {int. }}^{\mu \nu}$ is given by

$$
T_{\text {int. }}^{\mu \nu}=-\mathcal{M}^{\mu \lambda} F_{\lambda}^{\nu}=\left(\begin{array}{ccc}
\mathcal{M}^{t \theta} F_{t \theta} & 0 & -\mathcal{M}^{\theta \phi} F_{t \theta} \\
0 & -\ell^{-2}\left(\mathcal{M}^{t \theta} F_{t \theta}+\mathcal{M}^{\theta \phi} F_{\theta \phi}\right) & 0 \\
-\mathcal{M}^{\theta \phi} F_{t \theta} & 0 & -\ell^{-2} S^{-2}(\theta) \mathcal{M}^{\theta \phi} F_{\theta \phi}
\end{array}\right) .
$$

Eqns. (3.23) and (3.25) imply $F^{t}{ }_{\mu} J^{\mu}=F^{\phi}{ }_{\mu} J^{\mu}=0$, which are automatically satisfied if the susceptibility $\chi$ does not depend on $t$ and $\phi$, which we assume in the following. The only nontrivial equation of motion (3.24) becomes

$$
\frac{d \mathcal{P}}{d \theta}-(\mathcal{P}+\rho) \frac{d \ln \gamma}{d \theta}-\frac{1}{2} \mathcal{M}^{\nu \lambda} \nabla_{\theta} F_{\nu \lambda}=r F_{\theta \mu} u^{\mu} .
$$

Using the Gibbs-Duhem relation (2.19) as well as (2.25), equ. (3.38) can be cast into the form

$$
\gamma s \frac{d}{d \theta}\left(\frac{\mathcal{T}}{\gamma}\right)+r \gamma \frac{d}{d \theta}\left(\frac{\mu}{\gamma}\right)=r F_{\theta \mu} u^{\mu}
$$

which is automatically solved using (3.35). Therefore, the rigidly rotating configurations are stationary solutions of the magnetohydrodynamic equations. Moreover, as the diffusion current and the heat flux vanish, the only nonzero contributions to the transport R-charge- and entropy currents come from the perfect fluid pieces (3.26), which are easily seen to be conserved as well.

From the grandcanonical potential (2.2) we find

$$
\begin{aligned}
\mathcal{P} & =\mathcal{T}^{3} h(\nu, b), \quad \mathcal{M}=\mathcal{T} \frac{\partial h}{\partial b}, \quad s=\mathcal{T}^{2}\left(3 h-\nu \frac{\partial h}{\partial \nu}-2 b \frac{\partial h}{\partial b}\right), \\
\rho & =2(\mathcal{P}-\mathcal{M B})=2 \mathcal{T}^{3}\left(h-b \frac{\partial h}{\partial b}\right), \quad r=\mathcal{T}^{2} \frac{\partial h}{\partial \nu} .
\end{aligned}
$$


In the nonrotating case $\omega=0$, we have $\gamma=1, F_{\theta \sigma} u^{\sigma}=F_{\theta \phi} u^{\phi}=F_{\theta \phi} \gamma \omega=0, \mathcal{B}=B=$ const., and thus (3.35) implies that the local temperature $\mathcal{T}$ as well as $\nu$ and $b$ are constant. We can then easily compute the energy, entropy, R-charge and magnetization, with the result

$$
\begin{aligned}
E & =2 \tau^{3} V\left(h-b \frac{\partial h}{\partial b}\right), \quad R=\tau^{2} V \frac{\partial h}{\partial \nu}, \\
S & =\tau^{2} V\left(3 h-\nu \frac{\partial h}{\partial \nu}-2 b \frac{\partial h}{\partial b}\right), \quad M=\tau V \frac{\partial h}{\partial b} .
\end{aligned}
$$

It is straightforward to verify ${ }^{8}$ that the Hawking temperature of the black hole is given by $T=\tau$, and the chemical potential associated to the R-charge is $\zeta=\tau \nu$. Using the relations

$$
\frac{\partial h}{\partial \nu}=\frac{\sqrt{2} N^{3 / 2}}{6 \pi} H \nu, \quad \frac{\partial h}{\partial b}=-\frac{\sqrt{2} N^{3 / 2}}{6 \pi} \frac{b}{H},
$$

following from (3.19), (3.20), one easily shows that $E, S$ and $R$ coincide with the corresponding expressions (3.15), (3.12) and (3.11) for the static black hole.

In the next subsection we will show that, using the function $h$ determined from the static $\mathrm{AdS}_{4}$ black hole as an input into the MHD equations, one can exactly reproduce the thermodynamics, boundary stress tensor and R-current of the rotating dyonic Kerr-Newman- $\mathrm{AdS}_{4}$ solution.

\section{$3.2 \quad$ Kerr-Newman-AdS 4}

The Kerr-Newman-AdS 4 solution to the equations of motion following from (3.1) is given by ${ }^{9}$

$$
\begin{gathered}
d s^{2}=-\frac{\Delta_{r}}{\rho^{2}}\left[d t-\frac{a \sin ^{2} \theta}{\Xi} d \phi\right]^{2}+\frac{\rho^{2}}{\Delta_{r}} d r^{2}+\frac{\rho^{2}}{\Delta_{\theta}} d \theta^{2}+\frac{\Delta_{\theta} \sin ^{2} \theta}{\rho^{2}}\left[a d t-\frac{r^{2}+a^{2}}{\Xi} d \phi\right]^{2}, \\
A=-\frac{q_{e} r}{\rho^{2}}\left[d t-\frac{a \sin ^{2} \theta}{\Xi} d \phi\right]+\frac{q_{m} \cos \theta}{\rho^{2}}\left[a d t-\frac{r^{2}+a^{2}}{\Xi} d \phi\right],
\end{gathered}
$$

where

$$
\begin{gathered}
\Delta_{r}=\left(r^{2}+a^{2}\right)\left(1+\frac{r^{2}}{\ell^{2}}\right)-2 m r+q_{e}^{2}+q_{m}^{2}, \quad \Xi=1-\frac{a^{2}}{\ell^{2}}, \\
\rho^{2}=r^{2}+a^{2} \cos ^{2} \theta, \quad \Delta_{\theta}=1-\frac{a^{2}}{\ell^{2}} \cos ^{2} \theta,
\end{gathered}
$$

and $a$ is a rotation parameter.

The thermodynamical phase structure and stability of these black holes was studied in detail in [22]. In order to get the boundary geometry $d \sigma^{2}$, one takes $r=$ const. $\rightarrow \infty$, and then rescales $d s^{2}$ by $\ell^{2} / r^{2}$. This leads to

$$
d \sigma^{2}=-\left[d t-\frac{a \sin ^{2} \theta}{\Xi} d \phi\right]^{2}+\frac{\ell^{2}}{\Delta_{\theta}} d \theta^{2}+\frac{\ell^{2} \Delta_{\theta} \sin ^{2} \theta}{\Xi^{2}} d \phi^{2},
$$

which is a rotating Einstein universe. In the limit of large $r$, the $\mathrm{U}(1)$ field strength behaves as

$$
F \rightarrow \frac{q_{m}}{\Xi} \sin \theta d \theta \wedge d \phi
$$

\footnotetext{
${ }^{8}$ One can express $d E-\tau d S-\tau \nu d R+M d B$ in terms of $d \tau, d \nu, d b$ and check that it vanishes.

${ }^{9}$ For the time being, we restrict to the case of spherical horizons. The generalization to $k=0,-1$ is straightforward.
} 
As before, the expectation value of the CFT R-current $J^{\mu}$ can be computed by varying the action on-shell with respect to the gauge potential $A^{\mu}$ and subsequently rescaling by $r^{3} / \ell^{2}$. This yields

$$
J_{t}=-\frac{\sqrt{2} N^{3 / 2} q_{e}}{6 \pi \ell^{3}}, \quad J_{\phi}=\frac{\sqrt{2} N^{3 / 2} q_{e} a \sin ^{2} \theta}{6 \pi \ell^{3} \Xi}, \quad J_{\theta}=0 .
$$

The chemical potential $\zeta$ of the dual field theory is given by [22]

$$
\zeta \ell=\left.A_{\nu}^{e} \chi^{\nu}\right|_{r \rightarrow \infty}-\left.A_{\nu}^{e} \chi^{\nu}\right|_{r=r_{h}}=\frac{q_{e} r_{h}}{r_{h}^{2}+a^{2}},
$$

where $\chi=\partial_{t}+\Omega_{H} \partial_{\phi}$ denotes the null generator of the horizon and $A_{\nu}^{e}$ is the electric part of the vector potential. The angular velocity of the horizon reads

$$
\Omega_{H}=\frac{a \Xi}{r_{h}^{2}+a^{2}} .
$$

In what follows, we shall be interested in the limit of large black holes, $r_{h} \gg \ell$, when a hydrodynamical description is valid. As $a^{2}<\ell^{2}$ (otherwise $\Delta_{\theta}$ can become negative), we have then also $r_{h}^{2} \gg a^{2}$, and the chemical potential (3.50) reduces to

$$
\zeta=\frac{q_{e}}{\ell r_{h}} .
$$

The electric charge of the black hole (the field theory R-charge) is obtained by computing the flux of the electromagnetic field strength at infinity, with the result ${ }^{10}$

$$
R=\frac{\ell q_{e}}{\Xi G}
$$

The temperature, Bekenstein-Hawking entropy, energy and angular momentum are given by [22]

$$
\begin{aligned}
T & =\frac{r_{h}}{4 \pi\left(r_{h}^{2}+a^{2}\right)}\left(1+\frac{a^{2}}{\ell^{2}}+\frac{3 r_{h}^{2}}{\ell^{2}}-\frac{a^{2}+q_{e}^{2}+q_{m}^{2}}{r_{h}^{2}}\right) \approx \frac{1}{4 \pi r_{h}}\left(\frac{3 r_{h}^{2}}{\ell^{2}}-\frac{q_{e}^{2}+q_{m}^{2}}{r_{h}^{2}}\right), \\
S & =\frac{\pi\left(r_{h}^{2}+a^{2}\right)}{\Xi G} \approx \frac{2 \pi \sqrt{2} N^{3 / 2} r_{h}^{2}}{3 \ell^{2} \Xi}, \\
E & =\frac{m}{\Xi^{2} G} \approx \frac{\sqrt{2} N^{3 / 2}}{3 \ell^{2} \Xi^{2}}\left[\frac{r_{h}^{3}}{\ell^{2}}+\frac{q_{e}^{2}+q_{m}^{2}}{r_{h}}\right], \\
J & =a E,
\end{aligned}
$$

where in the last steps we took the large black hole limit. Let us write the metric (3.43) in the ADM form

$$
d s^{2}=-N^{2} d t^{2}+\frac{\rho^{2}}{\Delta_{r}} d r^{2}+\frac{\rho^{2}}{\Delta_{\theta}} d \theta^{2}+\frac{\Sigma^{2} \sin ^{2} \theta}{\rho^{2} \Xi^{2}}(d \phi-\Omega d t)^{2}
$$

where

$$
\Sigma^{2}=\left(r^{2}+a^{2}\right)^{2} \Delta_{\theta}-a^{2} \Delta_{r} \sin ^{2} \theta
$$

\footnotetext{
${ }^{10}$ Note that we rescaled $R$ by a factor of $\ell$ and $\zeta$ by $\ell^{-1}$ with respect to [22].
} 
and the lapse function $N$ and the angular velocity $\Omega$ are defined by

$$
\begin{aligned}
N^{2} & =\frac{\rho^{2} \Delta_{r} \Delta_{\theta}}{\Sigma^{2}}, \\
\Omega & =\frac{a \Xi}{\Sigma^{2}}\left[\Delta_{\theta}\left(r^{2}+a^{2}\right)-\Delta_{r}\right] .
\end{aligned}
$$

It was shown in [22] that the angular velocity $\omega$ entering the thermodynamics is

$$
\omega=\Omega_{H}-\Omega_{\infty}=\frac{a\left(1+r_{h}^{2} / \ell^{2}\right)}{r_{h}^{2}+a^{2}},
$$

which boils down to

$$
\omega \approx a / \ell^{2}
$$

for large black holes.

By applying the implicit coordinate transformation $[23]^{11}$

$$
T=t, \quad \Phi=\phi+\frac{a}{\ell^{2}} t, \quad y \cos \Theta=r \cos \theta, \quad y^{2}=\frac{1}{\Xi}\left[r^{2} \Delta_{\theta}+a^{2} \sin ^{2} \theta\right]
$$

to the solution (3.43), one gets a different slicing such that the boundary geometry is that of a static Einstein universe,

$$
d \tilde{\sigma}^{2}=-d T^{2}+\ell^{2}\left(d \Theta^{2}+\sin ^{2} \Theta d \Phi^{2}\right) .
$$

Note that (3.64) is related to (3.47) by a diffeomorphism plus a Weyl rescaling: On the boundary $r \rightarrow \infty$ (which implies $y \rightarrow \infty$ ), the coordinate transformation (3.63) reduces to

$$
T=t, \quad \Phi=\phi+\frac{a}{\ell^{2}} t, \quad \sin \Theta=\frac{\sin \theta}{\sqrt{\Delta_{\theta}}} .
$$

Applying this to (3.64) and subsequently rescaling with $e^{2 \varphi}=\Delta_{\theta} / \Xi$ yields (3.47). The two boundary metrics are thus conformally related. For the five-dimensional Kerr-AdS black hole, this was first noticed in [24].

In the new coordinates, the Faraday tensor on the boundary becomes

$$
F \rightarrow q_{m}\left(1-\frac{a^{2}}{\ell^{2}} \sin ^{2} \Theta\right)^{-3 / 2} \sin \Theta d \Theta \wedge\left(d \Phi-\frac{a}{\ell^{2}} d T\right)
$$

Due to the nonvanishing component $F_{\Theta T}$, we have now also an external electric field. This is not surprising; it results from the boost in (3.65).

In order to proceed, we need the thermodynamic variable $\mathcal{B}$. We already stated in section 2.2 that $\mathcal{B}^{2}$ is proportional to $F_{\mu \nu} F^{\mu \nu}$. To fix the prefactor, note that for the field strength (3.66), one gets

$$
F_{\mu \nu} F^{\mu \nu}=\frac{2 q_{m}^{2}}{\ell^{4}}\left(1-\frac{a^{2}}{\ell^{2}} \sin ^{2} \Theta\right)^{-2}
$$

\footnotetext{
${ }^{11}$ We apologize for using the same symbol $\Phi$ for an angular coordinate and the grandcanonical potential, but the meaning should be clear from the context.
} 
which reduces to $F^{2}=2 q_{m}^{2} / \ell^{4}=2 \ell^{2} \mathcal{B}^{2}$ in the static case $a=0$. We have thus ${ }^{12}$

$$
\mathcal{B}=\frac{\sqrt{F^{2}}}{\sqrt{2} \ell} .
$$

The grandcanonical potential $\Phi(\mathcal{T}, \mathcal{V}, \mu, \mathcal{B})$ is given by $(3.18)$, with $h(\nu, b)$ defined in (3.19), (3.20), and

$$
b=\frac{\mathcal{B}}{\mathcal{T}^{2}}=\frac{\sqrt{F^{2}}}{\sqrt{2} \ell \mathcal{T}^{2}} .
$$

One can then compute the polarization tensor $\mathcal{M}^{\mu \nu}$, with the result

$$
\mathcal{M}^{\mu \nu}=-\frac{1}{\mathcal{V}} \frac{\partial \Phi}{\partial F_{\mu \nu}}=\chi F^{\mu \nu}
$$

with the susceptibility

$$
\chi=\frac{1}{\ell^{2} b \mathcal{T}} \frac{\partial h}{\partial b}=-\frac{\sqrt{2} N^{3 / 2}}{6 \pi H \ell^{2} \mathcal{T}} .
$$

Note that $\chi$ is always negative, so that our fluid is diamagnetic. Moreover, for large (local) temperatures $\mathcal{T}$ (keeping $\mu$ and $\mathcal{B}$ finite), (3.20) gives $H\left(\mu / \mathcal{T}, \mathcal{B} / \mathcal{T}^{2}\right) \rightarrow 4 \pi / 3$, and thus

$$
\chi \rightarrow-\frac{C}{\mathcal{T}}, \quad C \equiv \frac{\sqrt{2} N^{3 / 2}}{8 \pi^{2} \ell^{2}},
$$

i. e., the susceptibility obeys a diamagnetic Curie law with Curie constant $C$.

Let us now consider the MHD equations $\nabla_{\mu} T^{\mu \nu}=F_{\mu}^{\nu} J^{\mu}$ with the field strength (3.66). One easily shows that $F^{\nu}{ }_{\mu} u^{\mu}=0$ for $\nu=T, \Phi$, and that $F^{\Theta}{ }_{\mu} u^{\mu}$ is proportional to $\omega-a / \ell^{2}$. Let us assume that this vanishes, i. e. , that the angular velocity of the fluid is given by $\omega=a / \ell^{2}$, as is suggested by (3.62). Then the transport current $r u^{\mu}$ is orthogonal to the electromagnetic field, and (3.35) implies that $\nu=\mu / \mathcal{T}$ is constant. Moreover, from (3.69) and (3.67) we have

$$
b=\frac{q_{m}}{\ell^{3} \mathcal{T}^{2}}\left(1-\frac{a^{2}}{\ell^{2}} \sin ^{2} \Theta\right)^{-1}=\frac{q_{m} \gamma^{2}}{\ell^{3} \mathcal{T}^{2}} \quad \text { for } \quad \omega=\frac{a}{\ell^{2}},
$$

which is constant by virtue of (3.35). Using this, it is straightforward to shew that the polarization current $J_{\text {micr. }}^{\mu}=-\nabla_{\sigma} \mathcal{M}^{\sigma \mu}$ is proportional to $u^{\mu}$ in this case, and hence orthogonal to $F^{\nu}{ }_{\mu}$ as well. This means that $F^{\nu}{ }_{\mu} J^{\mu}=0$; due to orthogonal magnetic and electric fields compensating each other, there is no net Lorentz force acting on the fluid, and the MHD equations boil down to $\nabla_{\mu} T^{\mu \nu}=0$. It is interesting to consider this situation from the point of view of a reference frame which is moving with the charged fluid (the rotating Einstein universe (3.47)). In this reference frame the fluid is static and therefore not subject to the magnetic force. Since the net electromagnetic force, which in this frame consists only of the electric force, must be zero, the electric field must vanish in the moving reference frame. This is indeed the case, as can be seen from (3.48).

\footnotetext{
${ }^{12} \mathrm{Up}$ to the prefactor $\ell$, that stems from the fact that the boundary Faraday tensor is obtained from the corresponding bulk quantity by projecting on the boundary and then rescaling with $\ell^{-1}$, in flat spacetime this would just be the statement that $F^{2}=2\left(\mathcal{B}^{2}-\mathcal{E}^{2}\right)$, and we defined $\mathcal{B}^{2}$ to be what is usually called $\mathcal{B}^{2}-\mathcal{E}^{2}$.
} 
It would be very interesting to see to which kind of black holes the fluid configurations with $\omega$ different from $a / \ell^{2}$ correspond to. They might have to do with a nontrivial nut parameter, but we shall leave this point for future investigations.

One can now proceed to compute the conserved charges. From (3.28) - (3.31) one obtains

$$
\begin{aligned}
& S=\frac{4 \pi \ell^{2} \tau^{2}}{\Xi}\left(3 h-\nu \frac{\partial h}{\partial \nu}-2 b \frac{\partial h}{\partial b}\right), \quad R=\frac{4 \pi \ell^{2} \tau^{2}}{\Xi} \frac{\partial h}{\partial \nu}, \\
& E=\frac{8 \pi \ell^{2} \tau^{3}}{\Xi^{2}}\left(h-b \frac{\partial h}{\partial b}\right), \quad J=\frac{8 \pi \omega \ell^{4} \tau^{3}}{\Xi^{2}}\left(h-b \frac{\partial h}{\partial b}\right),
\end{aligned}
$$

together with the magnetization

$$
M=\int d^{2} x \sqrt{-g} \mathcal{M} \gamma^{-1}=4 \pi \ell^{2} \tau \frac{\partial h}{\partial b} .
$$

The temperature $T$ and chemical potential $\zeta$ are given by $T=\tau$ and $\zeta=\tau \nu$ respectively, while the intensive variable conjugate to $M$ is $B=\tau^{2} b / \Xi$. To show this, one can express $d E-\tau d S-$ $\tau \nu d R+M d B-\omega d J$ in terms of $d \tau, d \nu, d b, d \omega$ and check that it vanishes. Note that $T, \zeta$ and $B$ are distinct from $\mathcal{T}, \mu$ and $\mathcal{B}$. While the former quantities are asssociated to the whole fluid configuration, the latter are local thermodynamic properties of the fluid that vary on the boundary manifold. For the grandcanonical partition function one finds

$$
\ln \mathcal{Z}_{g c}=-\frac{1}{T}(E-T S-\omega J-\zeta R)=\frac{V T^{2} h\left(\zeta / T, B \Xi / T^{2}\right)}{\Xi},
$$

which means that $\ln \mathcal{Z}_{g c}$ of the rotating fluid is obtained simply by dividing the grandcanonical partition function of the static fluid by the universal factor $\Xi=1-\omega^{2} \ell^{2}$, and replacing $B$ by $B \Xi$. This generalizes the results of [13] to nonvanishing magnetic fields.

We must now compare the fluid charges with the corresponding black hole quantities. To do this, we first note that for large KNAdS black holes, the temperature has the same dependence on $r_{h}$ as in the static case (compare (3.54) with (3.17)), so that the function $H=r_{h} /\left(\ell^{2} T\right)$ is again determined by (3.20). Using this together with (3.19) and (3.42), one easily shows that the fluid charges $R, S, E, J$ exactly coincide with the expressions for the black hole given in (3.53)-(3.57). This coincidence extends also to the energy-momentum tensor and the R-current: The holographic stress tensor of the KNAdS black hole was determined in [22], and reads

$$
\begin{gathered}
T_{t t}=\frac{2 m}{8 \pi G \ell^{2}}, \quad T_{t \phi}=-\frac{2 m a \sin ^{2} \theta}{8 \pi G \Xi \ell^{2}}, \\
T_{\phi \phi}=\frac{m \sin ^{2} \theta\left[\ell^{2}+3 a^{2} \sin ^{2} \theta-a^{2}\right]}{8 \pi G \Xi^{2} \ell^{2}}, \quad T_{\theta \theta}=\frac{m}{8 \pi G \Delta_{\theta}},
\end{gathered}
$$

and all other components vanishing. This corresponds to the boundary geometry (3.47), i. e. , to the rotating Einstein universe. To get the stress tensor for the static Einstein universe (3.64), recall that the latter is related to the former by a diffeomorphism plus a Weyl rescaling, $d \sigma^{2}=$ $e^{2 \varphi} d \tilde{\sigma}^{2}$, with $e^{2 \varphi}=\Delta_{\theta} / \Xi$. Under a Weyl rescaling, the energy-momentum tensor transforms as $T_{\mu \nu}=e^{-(d-2) \varphi} \tilde{T}_{\mu \nu}$ (see e. g. [13]), which yields (taking $d=3$ )

$$
\tilde{T}_{T T}=\frac{m \gamma^{3}}{8 \pi G \ell^{2}}\left(3 \gamma^{2}-1\right), \quad \tilde{T}_{T \Phi}=-\frac{3 m a \sin ^{2} \Theta}{8 \pi G \ell^{2}} \gamma^{5},
$$




$$
\tilde{T}_{\Phi \Phi}=\frac{m \sin ^{2} \Theta}{8 \pi G} \gamma^{3}\left(3 \gamma^{2}-2\right), \quad \tilde{T}_{\Theta \Theta}=\frac{m \gamma^{3}}{8 \pi G},
$$

which is easily shown to exactly coincide with the fluid stress tensor (3.36).

The R-current (3.49) transforms as $\tilde{J}^{\mu}=e^{-d \varphi} J^{\mu}$ under a Weyl rescaling [13]. This gives for the R-current corresponding to the static boundary geometry

$$
\tilde{J}^{T}=\gamma^{3} \frac{\sqrt{2} N^{3 / 2} q_{e}}{6 \pi \ell^{3}}, \quad \tilde{J}^{\Phi}=\frac{a}{\ell^{2}} \tilde{J}^{T}, \quad \tilde{J}^{\Theta}=0 .
$$

This is again equal to the transport current $J_{\text {macr. }}^{\mu}=r u^{\mu}$ of the fluid.

\subsection{Black strings in $\mathrm{AdS}_{5}$}

We now solve the Navier-Stokes equations on $\mathbb{R} \times S^{1} \times S^{2}, \mathbb{R} \times S^{1} \times \mathbb{R}^{2}$ or $\mathbb{R} \times S^{1} \times H^{2}$. This will yield predictions for black strings in $\mathrm{AdS}_{5}$, for which at present only partial results are known [25-31]. Since the function $h(\nu, b)$ entering (2.2) is unknown for $d=4, b \neq 0$, we shall consider hydrodynamics rather than MHD, i. e., we will restrict to the case of zero electromagnetic fields in this section.

The metric entering the MHD equations is

$$
d s^{2}=-d t^{2}+d z^{2}+\ell^{2}\left(d \theta^{2}+S(\theta)^{2} d \phi^{2}\right),
$$

where $S(\theta)$ was given in (3.4). The only nonzero Christoffel symbols are the ones in (3.22). For stationary, translationally invariant and axisymmetric configurations one has $\partial_{t} T^{\mu \nu}=\partial_{z} T^{\mu \nu}=$ $\partial_{\phi} T^{\mu \nu}=0$, and the Navier-Stokes equations reduce to $(3.23)-(3.25)$ (with $F_{\mu \nu}=0$ ) plus the additional condition

$$
\partial_{\theta} T^{\theta z}+\frac{S^{\prime}}{S} T^{\theta z}=0
$$

The entropy and the R-charges read

$$
\begin{aligned}
S & =\int d^{3} x \sqrt{-g} J_{S}^{t}=\int d z d \theta d \phi \ell^{2} S(\theta) s \gamma \\
R_{I} & =\int d^{3} x \sqrt{-g} J_{I}^{t}=\int d z d \theta d \phi \ell^{2} S(\theta) r_{I} \gamma .
\end{aligned}
$$

Now the Killing vectors of interest are $\partial_{t}$ (energy $E$ ), $\partial_{\phi}$ (angular momentum $J$ on the $S^{2}, \mathbb{R}^{2}$ or $H^{2}$ ), and $\partial_{z}$ (momentum $p$ along the string). The associated conserved charges are

$$
\begin{aligned}
E & =\int d z d \theta d \phi \ell^{2} S(\theta) T^{t t}, \\
J & =\int d z d \theta d \phi \ell^{4} S^{3}(\theta) T^{t \phi}, \\
p & =\int d z d \theta d \phi \ell^{2} S(\theta) T^{t z} .
\end{aligned}
$$

The shear tensor $\sigma^{\mu \nu}$, heat flux $q^{\mu}$ and diffusion currents $q_{I}^{\mu}$ must vanish on any stationary solution of fluid dynamics. The requirement $\sigma^{\mu \nu}=0$ means that the fluid motion should be just a rigid 
rotation. By an $\mathrm{SO}(3)$ transformation we can choose this rotation such that the 4 -velocity of the fluid is $u^{\mu}=\left(u^{t}, u^{z}, u^{\theta}, u^{\phi}\right)=\gamma\left(1, \omega_{1}, 0, \omega_{2}\right)$ for some constants $\omega_{1}, \omega_{2}$. From $u^{\mu} u_{\mu}=-1$ and $\gamma=\left(1-v^{2}\right)^{-1 / 2}$ one obtains then $v^{2}=\omega_{1}^{2}+\ell^{2} \omega_{2}^{2} S^{2}(\theta)$.

The equilibrium fluid flow is symmetric under translations of $t, z$ and $\phi$, so that all thermodynamic quantities depend only on $\theta$. We now evaluate the expansion, acceleration, shear tensor, heat flux and diffusion current, with the result

$$
\begin{aligned}
\vartheta=0, \quad a^{\mu} & =\left(0,0,-S(\theta) S^{\prime}(\theta) \gamma^{2} \omega_{2}^{2}, 0\right), \quad \sigma^{\mu \nu}=0, \\
q^{\mu} & =-\ell^{-2} \kappa \gamma\left(0,0, \frac{d}{d \theta}\left(\frac{\mathcal{T}}{\gamma}\right), 0\right), \\
q_{I}^{\mu} & =-\ell^{-2} D_{I J}\left(0,0, \frac{d}{d \theta}\left(\frac{\mu^{J}}{\mathcal{T}}\right), 0\right) .
\end{aligned}
$$

The requirement that $q^{\mu}$ and $q_{I}^{\mu}$ vanish implies that

$$
\mathcal{T}=\tau \gamma, \quad \mu^{I}=\mathcal{T} \nu^{I}
$$

with $\tau$ and $\nu^{I}$ constant. The conditions (3.93) determine all the thermodynamic quantities as a function of the coordinate $\theta$. We now want to shew that this configuration solves the Navier-Stokes equations. To this end, we proceed as in [13], and first notice that the dissipative part of the energy-momentum tensor vanishes once (3.93) is imposed, so that all nonzero contributions to the stress tensor result from the perfect fluid part, and read

$$
\begin{array}{r}
T^{\mu \nu}= \\
\gamma^{2}\left(\begin{array}{cccc}
\rho+v^{2} \mathcal{P} & (\rho+\mathcal{P}) \omega_{1} & 0 & (\rho+\mathcal{P}) \omega_{2} \\
(\rho+\mathcal{P}) \omega_{1} & \rho \omega_{1}^{2}+\mathcal{P}\left(1-g^{-2} \omega_{2}^{2} S^{2}(\theta)\right) & 0 & (\rho+\mathcal{P}) \omega_{1} \omega_{2} \\
0 & 0 & \gamma^{-2} g^{2} \mathcal{P} & 0 \\
(\rho+\mathcal{P}) \omega_{2} & (\rho+\mathcal{P}) \omega_{1} \omega_{2} & 0 & \rho \omega_{2}^{2}+\mathcal{P} g^{2} S^{-2}(\theta)\left(1-\omega_{1}^{2}\right)
\end{array}\right) .
\end{array}
$$

The only nontrivial equation of motion (3.24) becomes

$$
\frac{d \mathcal{P}}{d \theta}-(\mathcal{P}+\rho) \frac{d \ln \gamma}{d \theta}=0
$$

Using the Gibbs-Duhem relation (2.19), (3.94) can be cast into the form

$$
\gamma s \frac{d}{d \theta}\left(\frac{\mathcal{T}}{\gamma}\right)+r_{I} \gamma \frac{d}{d \theta}\left(\frac{\mu^{I}}{\gamma}\right)=0
$$

which is automatically solved using (3.93). Therefore, the rigidly rotating configurations are stationary solutions of the equations of fluid dynamics.

From the grandcanonical potential

$$
\Phi=-\mathcal{V} \mathcal{T}^{4} h\left(\nu^{I}\right)
$$

one obtains

$$
\rho=3 \mathcal{P}=3 \mathcal{T}^{4} h\left(\nu^{I}\right), \quad r_{I}=\mathcal{T}^{3} h_{I}\left(\nu^{J}\right), \quad s=\mathcal{T}^{3}\left(4 h-\nu^{I} h_{I}\right)
$$


with

$$
h_{I}=\frac{\partial h}{\partial \nu^{I}} .
$$

We saw in the previous section that for large $\mathrm{AdS}_{4}$ black holes, the function $h$ is insensitive to the curvature parameter $k$. The same is to be expected for large black strings in $\mathrm{AdS}_{5}$, so that we can infer the function $h\left(\nu^{I}\right)$ from the $k=0$ case, which is simply the $\mathrm{AdS}_{5}$ black hole with flat horizon. This yields [13]

$$
h\left(\nu^{I}\right)=2 \pi^{2} N^{2} \frac{\prod_{J}\left(1+\kappa^{J}\right)^{3}}{\left(2+\sum_{J} \kappa^{J}-\prod_{J} \kappa^{J}\right)^{4}},
$$

where the auxiliary parameters $\kappa^{I}$ are related to the $\nu^{I}$ by

$$
\nu^{I}=\frac{2 \pi \prod_{J}\left(1+\kappa^{J}\right)}{2+\sum_{J} \kappa^{J}-\prod_{J} \kappa^{J}} \frac{\sqrt{\kappa^{I}}}{1+\kappa^{I}} .
$$

The conserved charges corresponding to our fluid configurations are

$$
\begin{gathered}
E=L \ell^{2} \tau^{4} h\left(\nu^{I}\right)\left(4 K_{1,6}\left(\omega_{1}, \omega_{2}\right)-K_{1,4}\left(\omega_{1}, \omega_{2}\right)\right), \\
p=4 L \ell^{2} \tau^{4} \omega_{1} h\left(\nu^{I}\right) K_{1,6}\left(\omega_{1}, \omega_{2}\right), \quad J=4 L \ell^{2} \tau^{4} \omega_{2} h\left(\nu^{I}\right) K_{3,6}\left(\omega_{1}, \omega_{2}\right), \\
S=L \ell^{2} \tau^{3}\left(4 h-\nu^{I} h_{I}\right) K_{1,4}\left(\omega_{1}, \omega_{2}\right), \quad R_{I}=L \ell^{2} \tau^{3} h_{I}\left(\nu^{J}\right) K_{1,4}\left(\omega_{1}, \omega_{2}\right),
\end{gathered}
$$

where $L$ is the length of the $S^{1}$ parametrized by $z$, and we have defined the integrals

$$
K_{m, n}\left(\omega_{1}, \omega_{2}\right)=\int S^{m}(\theta) \gamma^{n} d \phi d \theta
$$

These integrals, which can be performed in terms of elementary functions, satisfy the relations

$$
\frac{\partial K_{m, n}}{\partial \omega_{1}}=n \omega_{1} K_{m, n+2}, \quad \frac{\partial K_{m, n}}{\partial \omega_{2}}=n \ell^{2} \omega_{2} K_{m+2, n+2}
$$

and

$$
-\ell^{2} \omega_{2}^{2} K_{m, n}=K_{m-2, n-2}-\left(1-\omega_{1}^{2}\right) K_{m-2, n} .
$$

The chemical potentials corresponding to the rotating/boosted fluid solutions are defined by

$$
T=\left(\frac{\partial E}{\partial S}\right)_{J, p, R_{I}}, \quad w=\left(\frac{\partial E}{\partial p}\right)_{S, J, R_{I}}, \quad \Omega=\left(\frac{\partial E}{\partial J}\right)_{S, p, R_{I}}, \quad \zeta^{I}=\left(\frac{\partial E}{\partial R_{I}}\right)_{S, J, p, R_{K}} .
$$

One easily verifies that ${ }^{13}$

$$
T=\tau, \quad w=\omega_{1}, \quad \Omega=\omega_{2}, \quad \zeta^{I}=\tau \mu^{I} .
$$

Using this, we find for the grandcanonical partition function

$$
\ln \mathcal{Z}_{g c}=-\frac{1}{T}\left(E-T S-w p-\Omega J-\zeta^{I} R_{I}\right)=L \ell^{2} \tau^{3} h\left(\nu^{I}\right) K_{1,4}\left(\omega_{1}, \omega_{2}\right) .
$$

\footnotetext{
${ }^{13}$ As before, one can express $d E-\tau d S-\tau \nu^{I} d R_{I}-\omega_{1} d p-\omega_{2} d J$ in terms of $d \tau, d \nu^{I}, d \omega_{1}, d \omega_{2}$ and check that it vanishes. In order to show this, one has to use the relations (3.105) and (3.106).
} 
In the $k=0$ case, the integration is trivial and $\mathcal{Z}_{g c}$ reads

$$
\ln \mathcal{Z}_{g c}=\frac{V \tau^{3} h\left(\nu^{I}\right)}{\left(1-\omega_{1}^{2}-\ell^{2} \omega_{2}^{2}\right)^{2}},
$$

where $V$ is the volume of the three-torus.

For $k=1$, we have

$$
\ln \mathcal{Z}_{g c}=\frac{1}{2} V \tau^{3} h\left(\nu^{I}\right)\left[\frac{1}{\left(1-\omega_{1}^{2}\right)\left(1-\omega_{1}^{2}-\ell^{2} \omega_{2}^{2}\right)}+\frac{\arctan \left(\frac{\ell \omega_{2}}{\sqrt{1-\omega_{1}^{2}-\ell^{2} \omega_{2}^{2}}}\right)}{\ell \omega_{2}\left(1-\omega_{1}^{2}-\ell^{2} \omega_{2}^{2}\right)^{3 / 2}}\right],
$$

where $V=4 \pi L \ell^{2}$ is the volume of the $S^{1} \times S^{2}$. Note that this reduces, for $\omega_{1}=0$, to

$$
\ln \mathcal{Z}_{g c}=\frac{1}{2} V \tau^{3} h\left(\nu^{I}\right)\left[\frac{1}{1-\ell^{2} \omega_{2}^{2}}+\frac{\arctan \left(\frac{\ell \omega_{2}}{\sqrt{1-\ell^{2} \omega_{2}^{2}}}\right)}{\ell \omega_{2}\left(1-\ell^{2} \omega_{2}^{2}\right)^{3 / 2}}\right]
$$

while for $\omega_{2}=0$ it becomes

$$
\ln \mathcal{Z}_{g c}=\frac{1}{2} V \tau^{3} h\left(\nu^{I}\right)\left[\frac{1}{\left(1-\omega_{1}^{2}\right)^{2}}+1\right] .
$$

We see that the formula correctly reproduces the $1 /\left(1-\omega^{2}\right)$ behaviour characteristic of the rotation on spheres and the $1 /\left(1-\omega^{2}\right)^{2}$ behaviour of the boost along a circle (compare also with the results of [13] for Kerr-AdS black holes and with [23]).

Our results of this section yield predictions for rotating black strings in $\mathrm{AdS}_{5}$ with momentum along the string, that carry three electric charges (more precisely, there are electric charge densities along the string). While the general solutions of this type are still to be discovered, there are some partial numerical results available, namely for static charged black strings with all three charges equal [29]. It would be very interesting to compare the thermodynamics of these solutions with our hydrodynamical predictions. Unfortunately, the gravity solutions have been constructed only numerically, so that the comparison involves a fair amount of numerical work that goes beyond the scope of this paper.

\section{Gregory-Laflamme on magnetized plasma tubes}

\subsection{Description of the problem. Scherk-Schwarz compactification of a CFT}

It was observed in $[2,32,33]$ that, in the long wavelength regime, $d$-dimensional fluid dynamics is also an effective theory describing the Scherk-Schwarz (SS) compactification of a $(d+1)$-dimensional CFT. This theory is dual to SS compactification of $\mathrm{AdS}_{d+2}$ gravity. At finite temperature the theory has two vacuum solutions, namely i) the black brane solution that describes the deconfined phase of the dual gauge theory and ii) the AdS soliton that describes the confined phase [32]. In the 
neighborhood of the critical phase transition temperature the two phases can cohabit in equilibrium with a domain wall interface. Black holes in SS compactified $\mathrm{AdS}_{d+2}$ are then dual to deconfined plasma configurations immersed in the confined phase. The phase diagram of plasma equilibrium solutions includes plasma balls, pinched plasma balls, plasma rings and plasma tubes $[2,6,33]$. These plasma solutions are dual to SS compactified AdS black objects whose explicit solution is not yet known. However, quite interestingly, these phase diagrams reveal to have properties that are quite similar (although with some differences linked to the AdS nature of the system) to the known phase diagram solutions of asymptotically flat black holes. In [6] we have argued that this is not a coincidence since asymptotically flat black holes may indeed admit a fluid description in the limit of large number of dimensions. It is therefore important to explore the properties of plasma objects since they might provide a lighthouse to understand better even asymptotically flat black holes. We will return to this discussion in the end of this section.

In a previous paper [6], we have shown that plasma tubes suffer from the long wavelength Rayleigh-Plateau instability that makes them pinch-off when their length is bigger than their radius. We have further observed that these solutions are the natural duals of SS AdS black strings and that the plasma instability is dual to the Gregory-Laflamme instability of a black string. The aforementioned analysis was constructed on the top of a series of studies [34-39] where the remarkable analogy between fluid tubes and black strings was observed and analyzed. After [2,33], [6] promoted this curious analogy to an actual duality.

In this section we want to go a step forward and discuss the effect that a magnetic background introduces in the Rayleigh-Plateau instability of a plasma tube. This will provide solid predictions for the features of the dual magnetic black strings, associated Gregory-Laflamme instability and phase diagram of associated magnetically charged solutions.

We will take our boundary geometry to be $\mathbb{R}_{t} \times \mathbb{R}^{2} \times S^{1}$, described by the metric

$$
d s^{2}=-d t^{2}+d R^{2}+R^{2} d \phi^{2}+d z^{2},
$$

In this background, we consider fluid configurations that are uniform plasma tubes with topology $S^{1} \times D^{2}$, i.e., a disk extended along the periodic $z$-direction and immersed in the vacuum or confined phase. We also take them to be translationally invariant along the SS direction (not represented in (4.1)). Furthermore, we assume that there is a constant magnetic field along the $z$ direction. The dynamics of this plasma configuration is governed by the equations of relativistic magnetohydrodynamics (MHD) in $(3+1)$-dimensions. As mentioned above, in the long wavelength regime, MHD is an effective theory describing the SS compactification of a $(4+1)$-dimensional CFT. Moreover, in the dual gravity description these plasma tube configurations correspond to black strings in a background that asymptotes to a SS compactification of $\mathrm{AdS}_{6}$. The horizon topology of these strings is $S^{1} \times S^{3}$. The reason being that on the interface between the plasma phase and the vacuum, the SS circle shrinks to zero size as we enter in the holographic direction and reach the horizon radius. 


\subsection{MHD for Scherk-Schwarz plasmas}

In this subsection we find the MHD equations that describe the long wavelength magnetohydrodynamic limit of a Scherk-Schwarz compactification of a $(d+1)$-dimensional CFT. In the end we specialize to the $d=4$ case.

Neglecting subleading dissipation and diffusion contributions (in particular, in equilibrium these contributions vanish) the energy-momentum tensor of the fluid in the presence of an external electromagnetic field is the sum of the perfect fluid, Maxwell interaction and boundary surface contributions, ${ }^{14}$

$$
\begin{gathered}
T^{\mu \nu}=T_{\text {perf }}^{\mu \nu}+T_{\text {int }}^{\mu \nu}+T_{\text {bdry }}^{\mu \nu} ; \\
T_{\text {perf }}^{\mu \nu}=(\rho+\mathcal{P}) u^{\mu} u^{\nu}+\mathcal{P} g^{\mu \nu}, \\
T_{\text {int }}^{\mu \nu}=-\mathcal{M}^{\mu \lambda} F_{\lambda}^{\nu}, \\
T_{\text {bdry }}^{\mu \nu}=-\sigma h^{\mu \nu}|\partial f| \delta(f) .
\end{gathered}
$$

Here, $u^{\mu}$ is the fluid velocity, $\rho, \mathcal{P}$ and $\sigma$ are the density, pressure and surface tension of the fluid, $F_{\mu \nu}$ is the external Maxwell field and $\mathcal{M}^{\mu \nu}$ is the associated polarization tensor. The fluid boundary is defined by $f\left(x^{\mu}\right)=0$, it has unit normal $n_{\mu}=\partial_{\mu} f /|\partial f|$, and $h^{\mu \nu}=g^{\mu \nu}-n^{\mu} n^{\nu}$ is the projector onto the boundary.

The MHD equations follow from the covariant divergence of the stress tensor,

$$
\nabla_{\nu} T^{\mu \nu}=J_{\nu} F^{\mu \nu}
$$

where $J_{\nu}$ is the charge current defined in (2.7). From these equations we can derive the relativistic continuity, Navier-Stokes and Young-Laplace equations. In the absence of an electromagnetic field this derivation was presented in detail in [6]. With the extra Maxwell contribution the derivation follows similarly and we get (using footnote 2)

$$
\begin{aligned}
& u^{\mu} \partial_{\mu} \rho+(\rho+\mathcal{P}) \nabla_{\mu} u^{\mu}+\frac{1}{2} u^{\mu} \mathcal{M}^{\alpha \beta} \nabla_{\mu} F_{\alpha \beta}=0, \\
& (\rho+\mathcal{P}) u^{\nu} \nabla_{\nu} u^{\mu}=-\Pi^{\mu \nu}\left(\nabla_{\nu} \mathcal{P}-\frac{1}{2} \mathcal{M}^{\alpha \beta} \nabla_{\nu} F_{\alpha \beta}\right)+r u_{\nu} F^{\mu \nu}, \\
& \Pi_{<}-\Pi_{>}=\sigma K, \quad \Pi \equiv \mathcal{P}-\mathcal{M}^{\mu \alpha} F_{\nu \alpha} n_{\mu} n^{\nu}, \quad K \equiv h_{\mu}{ }^{\nu} \nabla_{\nu} n^{\mu},
\end{aligned}
$$

where $K$ is the boundary's extrinsic curvature and $\Pi$ is the generalized fluid pressure. It is the sum of the usual pressure $\mathcal{P}$ and the magnetic pressure (that is due to the Lorentz force exerted by the electromagnetic field on the polarization current). $\Pi_{<}-\Pi_{>}$is the generalized pressure jump when we cross the boundary from the exterior (with pressure $\Pi_{>}$) into the interior (with pressure $\left.\Pi_{<}\right)$. In the derivation of (4.6), the constraint that the fluid velocity must be orthogonal to the boundary normal is used (this guarantees that the fluid is confined inside the boundary),

$$
u^{\mu} n_{\mu}=0 .
$$

\footnotetext{
${ }^{14}$ The perfect fluid and Maxwell interaction terms were already introduced in (2.7). In section 2, where we studied the MHD equations of a $\mathrm{CFT}$ on $\mathbb{M}^{d}$, these were the only non-dissipative contributions. Here we want to study them in the case where we have a SS compactification of a $(4+1)$-dimensional CFT. Then there is also a surface contribution. It arises because in this case we have an interface between the plasma and the vacuum phases.
} 
Equations (4.4)-(4.7) constitute the set of relativistic MHD equations governing the fluid dynamics. A particular solution of these equations describes a static uniform plasma tube in the background (4.1) with radius $R_{0}$ and extended along the $z$-direction, and with a constant magnetic field $\mathcal{B}_{(0)}$ in the same direction. This solution is characterized by

$$
u_{(0)}^{\mu}=\delta_{t}^{\mu}, \quad \mathcal{B}_{(0)}^{\mu}=\mathcal{B}_{(0)} \delta_{z}^{\mu}, \quad \mathcal{P}=\mathcal{P}_{(0)}, \quad \rho=\rho_{(0)} .
$$

So, the non-vanishing Maxwell tensor components are $F_{R \phi}=-F_{\phi R}=\mathcal{B}_{(0)} \sqrt{-g}$ and $F_{\mu \nu} F^{\mu \nu}=$ $2 \mathcal{B}_{(0)}^{2}$. We use the subscript (0) to emphasize that this is a static unperturbed solution of MHD. In these conditions some terms in (4.4)-(4.6) simplify considerably. In particular, $\mathcal{M}^{\alpha \beta} \nabla_{\nu} F_{\alpha \beta}=$ $2 \mathcal{M}_{(0)} \nabla_{\nu} \mathcal{B}_{(0)}=0, r u_{\nu} F^{\mu \nu}=0$ and $\Pi=\mathcal{P}-\mathcal{M}_{(0)} \mathcal{B}_{(0)}\left(\right.$ where $\left.\mathcal{M}_{(0)}=\chi \mathcal{B}_{(0)}\right) . \mathcal{P}_{(0)}$ is then a constant by the Navier-Stokes equations (4.5). The pressure $\mathcal{P}_{(0)}$, energy density $\rho_{(0)}$ and magnetic field $\mathcal{B}_{(0)}$ are not independent. They are related through the appropriate equation of state for a SS compactification of a $(4+1)$-dimensional CFT. We derive this equation in the next subsection. It is given by (4.16) with $d=4$. This equation of state together with the continuity equation (4.4) demands that $\rho_{(0)}$ is also a constant.

In subsection 4.4 we will perturb equations (4.4)-(4.7) and study the Rayleigh-Plateau instability of a relativistic plasma tube in the presence of a magnetic field.

\subsection{Equation of state for a Scherk-Schwarz plasma}

In this subsection we discuss the grandcanonical potential and equation of state of a Scherk-Schwarz compactification of a $(d+1)$-dimensional CFT. The $d=4$ result will be needed in the next subsection.

Start with a $(d+1)$-dimensional CFT on $\mathbb{M}^{d} \times \mathcal{S}^{1}$, where $\mathcal{S}^{1}$ is the SS circle, in the presence of a conserved R-charge density $r$ (and associated chemical potential $\mu$ ) and of a constant magnetic field $\mathcal{B}$. Conformal invariance and extensivity demand that the grandcanonical potential,

$$
\Phi(\mathcal{T}, \mathcal{V}, \mu, \mathcal{B})=\mathcal{E}-\mathcal{T} \mathcal{S}-\mu \mathcal{R}
$$

is given by

$$
\Phi=-\mathcal{V} \mathcal{T}^{d+1} h(\nu, b),
$$

where we defined $\nu=\mu / \mathcal{T}$ and $b=\mathcal{B} / \mathcal{T}^{2}$. So, a CFT on $\mathbb{M}^{d} \times \mathcal{S}^{1}$ has the same thermodynamical potential as a CFT on $\mathbb{M}^{d+1}$ (that we analyzed in section $2.1^{15}$ ). The compact SS direction does however introduce a Casimir or vacuum energy density $\rho_{0}{ }^{16}$. Therefore, the equation of state of the $\mathrm{CFT}$ on $\mathbb{M}^{d} \times \mathcal{S}^{1}$ is

$$
\rho=d \mathcal{P}-2 \mathcal{B M}+\rho_{0}
$$

It follows from $\Phi=\Phi(\mathcal{T}, \mathcal{V}, \mu, \mathcal{B})$ and (4.10) that the pressure of the conformal fluid on $\mathbb{M}^{d+1}$ is given by

$$
\mathcal{P}=-\left.\frac{\partial \Phi}{\partial \mathcal{V}}\right|_{\mathcal{T}, \mu, \mathcal{B}}=\mathcal{T}^{d+1} h(\nu, b) .
$$

\footnotetext{
${ }^{15}$ Note that in section 2.1 we worked in $d$-dimensions while in this section we work in $d+1$ dimensions.

${ }^{16}$ In what follows, we shall assume that $\rho_{0}$ is independent of $\nu$ and $b$.
} 
Replacing this in the equation of state (4.11) we obtain for the local temperature

$$
\mathcal{T}=\left(\frac{\rho-\rho_{0}+2 \mathcal{B M}}{d h(\nu, b)}\right)^{\frac{1}{d+1}} .
$$

We can now do a dimensional reduction along the SS direction. This yields a field theory (that is not conformal) and which has a grandcanonical potential given by

$$
\begin{aligned}
\Phi_{\text {red }}(\mathcal{T}, \mathcal{V}, \mu, \mathcal{B}) & =\mathcal{E}-\mathcal{T} \mathcal{S}-\mu \mathcal{R} \\
& =\mathcal{V}\left(\rho_{0}-\mathcal{T}^{d+1} h(\nu, b)\right)
\end{aligned}
$$

Upon this reduction, the local temperature of the plasma does not change and is still given by (4.13). From $\Phi_{\text {red }}=\Phi_{\text {red }}(\mathcal{T}, \mathcal{V}, \mu, \mathcal{B})$ one has,

$$
\begin{aligned}
& s=-\frac{1}{\mathcal{V}} \frac{\partial \Phi_{\text {red }}}{\partial \mathcal{T}}=\mathcal{T}^{d}\left[(d+1) h(\nu, b)-\nu \partial_{\nu} h(\nu, b)-2 b \partial_{b} h(\nu, b)\right] \\
& \mathcal{P}=-\frac{\partial \Phi_{\text {red }}}{\partial \mathcal{V}}=\mathcal{T}^{d+1} h(\nu, b)-\rho_{0}, \\
& r=-\frac{1}{\mathcal{V}} \frac{\partial \Phi_{\text {red }}}{\partial \mu}=\mathcal{T}^{d} \partial_{\nu} h(\nu, b), \\
& \mathcal{M}=-\frac{1}{\mathcal{V}} \frac{\partial \Phi_{\text {red }}}{\partial \mathcal{B}}=\mathcal{T}^{d-1} \partial_{b} h(\nu, b) .
\end{aligned}
$$

Replacing (4.13) in the expression (4.15) for the pressure we get the equation of state,

$$
\mathcal{P}=\frac{\rho-(d+1) \rho_{0}+2 \mathcal{B M}}{d}
$$

which is valid in or out of equilibrium. A similar replacement could be done for the other equations in (4.15).

It is important to observe that the hydrodynamical equilibrium of the magnetized uniform tube also implies its thermodynamic equilibrium [6]. Indeed, the static condition $\partial_{\mu} \mathcal{P}=0$ that follows from the Navier-Stokes equation, together with the Gibbs-Duhem relation (2.19), written as $\nabla_{\nu} \mathcal{P}=s \nabla_{\nu} \mathcal{T}+r \nabla_{\nu} \mu+\mathcal{M} \nabla_{\nu} \mathcal{B}$, implies that the local temperature $\mathcal{T}$ is constant. Our uniform tube is static and thus the plasma temperature $T$ equals the local temperature $\mathcal{T}$. Demanding then that (4.13) is constant we find the relation (for constant $c$ )

$$
\rho-\rho_{0}=c d h(\nu, b)-2 \mathcal{B M}
$$

for an equilibrium static solution. The two last relations will be used later in the $d=4$ case.

\subsection{Rayleigh-Plateau instability in a magnetic background}

We now address the stability of a uniform plasma tube with a constant magnetic field along the tube direction when we perturb it. The dynamics of the perturbations are dictated by the MHD equations subject to appropriate boundary conditions. Once perturbations settle in, the static

plasma tube is taken away from thermal equilibrium and viscosity and diffusion effects start being 
also active. Therefore, the energy-momentum tensor of the fluid includes now not only the perfect fluid, the Maxwell interaction and the boundary surface tension terms (4.2), but also a dissipative contribution. The uniform plasma tube is afflicted by the Rayleigh-Plateau instability [6]. Surface tension is the mechanism responsible for this instability. Viscosity and diffusion play no role on the activation of the instability. Therefore, in our analysis we will neglect the dissipation contribution to the fluid stress tensor, and comment at the end on the effects it introduces. Our main aim is to find the effect that a magnetic field introduces on the instability, extending the analysis of [6].

Take a static uniform plasma tube with radius $R_{0}$ in a constant magnetic field $\mathcal{B}_{(0)}$ along the tube $z$-direction. This unperturbed solution is described by (4.8) and by the equation of state (4.16), with $d=4$.

Consider now perturbations on this plasma tube. A generic perturbation is described as

$$
u^{\mu}=u_{(0)}^{\mu}+\delta u^{\mu}, \quad \mathcal{P}=\mathcal{P}_{(0)}+\delta \mathcal{P}, \quad \rho=\rho_{(0)}+\delta \rho, \quad \mathcal{B}^{\mu}=\mathcal{B}_{(0)}^{\mu},
$$

where the perturbation on a quantity $Q$ is generically denoted by $\delta Q$. Note that the magnetic field is kept fixed. As there are no Maxwell equations on the boundary, it is treated as a non-dynamical parameter. Some of these perturbations are not independent. They are related by the equation of state (4.16), valid also out of equilibrium. Its perturbation yields the relation between the density and pressure perturbations,

$$
\delta \rho \simeq 4 \delta \mathcal{P} .
$$

In deriving this, we made the assumption that $\delta(\mathcal{B M})=\mathcal{B}^{2} \delta \chi$ vanishes. This seems reasonable for small magnetic fields, or in a regime where the susceptibility varies only slowly as a function of the temperature and the other thermodynamic variables. We shall comment later on possible effects that a relaxation of this assumption might have.

The perturbed state must satisfy the continuity and Navier-Stokes equations, (4.4) and (4.5). The Young-Laplace equation (4.6) and the constraint (4.7) provide boundary conditions for the perturbed problem. After eliminating the $0^{\text {th }}$ order terms using the unperturbed MHD equations, the continuity and Navier-Stokes eqns. yield, up to first order in the perturbation,

$$
\begin{aligned}
& \partial_{R} \delta u^{R}+\frac{1}{R} \delta u^{R}+\partial_{\phi} \delta u^{\phi}+\partial_{t} \delta u^{t}+\frac{1}{\rho_{(0)}+\mathcal{P}_{(0)}} \partial_{t} \delta \rho=0 \\
& \left(\rho_{(0)}+\mathcal{P}_{(0)}\right) \partial_{t} \delta u^{\mu}=-\delta^{\mu}{ }_{t} \partial_{t} \delta \mathcal{P}-g^{\mu \nu} \partial_{\nu} \delta \mathcal{P}+r \delta u_{R} F^{\mu R}+r \delta u_{\phi} F^{\mu \phi}=0 .
\end{aligned}
$$

Since any perturbation can be written as a Fourier series, in the most general case we will consider a perturbation that disturbs the boundary surface of the plasma tube according to

$$
R=R(t, z, \phi), \quad R(t, z, \phi)=R_{0}+\epsilon e^{\omega t} e^{i k z+i m \phi}, \quad \epsilon \ll R_{0},
$$

Positive $\omega$ describes an instability with wavenumber $k$. The possible unstable mode is axisymmetric if $m=0$. Naturally, we look for perturbations of the fluid quantities that have the same form as the boundary disturbance,

$$
\delta Q(t, R, z, \phi)=\delta Q(R) e^{\omega t} e^{i k z+i m \phi}, \quad \delta Q \equiv\left\{\delta u^{\mu}, \delta \mathcal{P}, \delta \rho\right\}
$$

which determines $\partial_{\mu} \delta Q$ for $\mu=t, z, \phi$. 
Solving (4.21) with perturbations (4.23) and (4.19) we find that the velocity perturbations at leading order are

$$
\begin{aligned}
& \delta u^{t}(R)=0 \\
& \delta u^{R}(R)=-\left[\omega\left(\rho_{(0)}+\mathcal{P}_{(0)}\right)\left(1+\frac{k_{A}^{2}}{k^{2}}\right)\right]^{-1}\left(\frac{d \delta \mathcal{P}(R)}{d R}+\frac{i m k_{A}}{k R} \delta \mathcal{P}\right), \\
& \delta u^{\phi}(R)=-\left[\omega\left(\rho_{(0)}+\mathcal{P}_{(0)}\right)\left(1+\frac{k_{A}^{2}}{k^{2}}\right)\right]^{-1}\left(-\frac{k_{A}}{k R} \frac{d \delta \mathcal{P}(R)}{d R}+\frac{i m}{R^{2}} \delta \mathcal{P}(R)\right), \\
& \delta u^{z}(R)=-i \frac{k}{\omega\left(\rho_{(0)}+\mathcal{P}_{(0)}\right)} \delta \mathcal{P}(R),
\end{aligned}
$$

where we defined the Alfvén wavenumber of the system,

$$
k_{A} \equiv \frac{\mathcal{B}_{(0)} r}{\rho_{(0)}+\mathcal{P}_{(0)}} \frac{k}{\omega} .
$$

Note that (4.24) satisfies the perturbed version of $u^{\mu} u_{\mu}=-1$, namely $u_{\mu}^{(0)} \delta u^{\mu}=0$.

Replacing (4.24) in the continuity equation (4.20) and using (4.19) we get

$$
\frac{d^{2} \delta \mathcal{P}(R)}{d R^{2}}+\frac{1}{R} \frac{d \delta \mathcal{P}(R)}{d R}-\left[\eta^{2}+\frac{m^{2}}{R^{2}}\right] \delta \mathcal{P}(R)=0, \quad \eta^{2} \equiv k^{2}\left(1+\frac{4 \omega^{2}}{k^{2}}\right)\left(1+\frac{k_{A}^{2}}{k^{2}}\right) .
$$

This is a modified Bessel equation. Its solutions are the modified Bessel functions of the first kind, $I_{m}(\eta R)$, and second kind $K_{m}(\eta R) . K_{m}(\eta R)$ diverges as $R^{-m}$ as $R \rightarrow 0$ and we discard it (for $m=0$, this solution would give a regular $\delta \mathcal{P}$ but $\delta u^{\mu}$ would still diverge; so we drop it also in this case). Therefore, the regular solution of (4.26) at the origin is

$$
\delta \mathcal{P}(R)=A I_{m}(\eta R)
$$

where $A$ is a constant to be fixed. Replacing the pressure perturbation in (4.24) yields for the radial component of the velocity,

$$
\delta u^{R}(R)=-\frac{A \eta}{\omega\left(\rho_{(0)}+\mathcal{P}_{(0)}\right)\left(1+\frac{k_{A}^{2}}{k^{2}}\right)}\left(I_{m}^{\prime}(\eta R)+\frac{i m}{\eta R} \frac{k_{A}}{k} I_{m}(\eta R)\right),
$$

where $I_{\nu}^{\prime}(y) \equiv \partial_{y} I_{\nu}(y)$.

The solutions just found must satisfy a total of two appropriate boundary conditions. The first demands normal stress balance on the boundary. This means that the generalized pressure perturbation must also satisfy the third perturbed hydrodynamic equation, namely the one that follows from perturbing the Young-Laplace ewquation (4.6),

$$
\mathrm{BC} \mathrm{I}:\left.\quad \delta \Pi\right|_{\mathrm{bdry}} \simeq \sigma\left[\left.K\right|_{R(t, \phi, z)}-\left.K\right|_{R_{0}}\right] .
$$

Here, $\left.\left.\delta \Pi\right|_{\text {bdry }} \simeq \delta \mathcal{P}\right|_{\text {bdry }}$ up to order $\epsilon^{2}$. The reason being that the external magnetic field is 
non-dynamical and thus fixed and the perturbation on the boundary normal is of order $\epsilon^{2} .{ }^{17}$ On the rhs we evaluate the expression at the perturbed boundary $R=R(t, \phi, z)$ defined in (4.22) and we subtract the unperturbed contribution evaluated at $R=R_{0}$. The extrinsic curvature is obtained from its definition, $K=h_{\mu}{ }^{\nu} \nabla_{\nu} n^{\mu}$, with the unit normal of the boundary (4.22), $n_{\mu}=$ $|n|^{-1}\left(-R_{t}^{\prime} \delta_{\mu}^{t}+\delta_{\mu}^{R}-R_{\phi}^{\prime} \delta_{\mu}^{\phi}-R_{z}^{\prime} \delta_{\mu}^{z}\right)$. This condition fixes the ratio $A / \epsilon$ to leading order as

$$
\frac{A}{\epsilon} \simeq \frac{\sigma}{R_{0}^{2} I_{m}\left(\eta R_{0}\right)}\left(k^{2} R_{0}^{2}+\omega^{2} R_{0}^{2}-1+m^{2}\right)
$$

The second boundary condition is a kinematic condition requiring that the normal component of the fluid velocity on the boundary satisfies the perturbed version of (4.7), $u_{(0)}^{\mu} \delta n_{\mu}+\delta u^{\mu} n_{\mu}^{(0)}=0$, where $\left.\delta n_{\mu} \equiv n_{\mu}\right|_{R(t, z, \phi)}-n_{\mu}^{(0)}$ and the unperturbed normal is $\left.n_{\mu}^{(0)} \equiv n_{\mu}\right|_{R_{0}}=\delta_{\mu}^{R}$. This ensures that the velocity perturbation leaves the fluid confined inside the boundary. This boundary condition then reads

$$
\left.\mathrm{BC} \mathrm{II} \mathrm{:} \quad \delta u^{R}\right|_{\mathrm{bdry}} \simeq \omega \epsilon e^{\omega t} e^{i m \phi+i k z},
$$

where the lhs follows from (4.28) evaluated at the boundary. This boundary condition together with (4.30) yields the desired dispersion relation $\omega(k)$ for the Rayleigh-Plateau instability in the presence of a constant magnetic field along the plasma tube direction,

$$
\omega^{2}=\frac{\sigma}{\left(\rho_{(0)}+\mathcal{P}_{(0)}\right) R_{0}^{3}}\left[\frac{k R_{0} I_{m+1}\left(\eta R_{0}\right)}{I_{m}\left(\eta R_{0}\right)}+m\left(1+i \frac{k_{A}}{\eta}\right)\right]\left(1+\frac{k_{A}^{2}}{k^{2}}\right)^{-\frac{1}{2}}\left(1-m^{2}-\omega^{2} R_{0}^{2}-k^{2} R_{0}^{2}\right),
$$

where we used the relation $I_{\nu}^{\prime}(y)=I_{\nu+1}(y)+\frac{\nu}{y} I_{\nu}(y)$ and $\eta$ is defined in (4.26). $\rho_{(0)}+\mathcal{P}_{(0)}$ is obtained from the equation of state (4.16) and (4.17) for $d=4$.

We conclude that the Rayleigh-Plateau instability is active $(\omega>0)$ for wavenumbers $k$ satisfying the condition

$$
k \leqslant \frac{\sqrt{1-m^{2}}}{R_{0}} .
$$

Since $m$ is an integer this means that only axisymmetric modes $(m=0)$ are unstable. In a previous paper [6] we have shown that the condition (4.33) for the threshold wavenumber can be found by noting that unstable modes are those that reduce the potential energy of the system for fixed volume (equivalently, they are those that maximize the entropy for fixed conserved charges). In the present system we have a competition between the surface tension and magnetic potential energies. However, to leading order $\mathcal{O}(\epsilon)$ in the perturbation, the magnetic energy does not change. Indeed, the magnetic field is kept fixed without being dynamically perturbed, see (4.18), and the volume change that also contributes to the magnetic energy variation is subleading, i.e., $\mathcal{O}\left(\epsilon^{2}\right)$ (see footnote 17 ), when compared with the $\mathcal{O}(\epsilon)$ change in the surface tension energy. Therefore, only the surface

\footnotetext{
${ }^{17}$ This point deserves a closer look. One has $\left.\delta \Pi\right|_{\text {bdry }}=\left.\delta \mathcal{P}\right|_{\text {bdry }}-\left.\delta\left(\mathcal{M}^{\mu \alpha} F_{\alpha}^{\nu} n_{\mu} n_{\nu}\right)\right|_{\text {bdry }}$; see (4.6). Assuming $\delta \chi \sim 0$, consistent with the approximation taken in (4.19), the magnetic contribution can be written as $\mathcal{M}^{\mu \alpha} F_{\alpha}^{\nu} \delta\left(n_{\mu} n_{\nu}\right)=\mathcal{M}^{\mu \alpha} F_{\alpha}^{\nu}\left(n_{\mu}^{(0)} \delta n_{\nu}+\delta n_{\mu} n_{\nu}^{(0)}\right)$, where $\delta n_{\mu}=\left.n_{\mu}\right|_{R(t, \phi, z)}-n_{\mu}^{(0)}$ and $n_{\mu}^{(0)}=\left.n_{\mu}\right|_{R_{0}}=\delta_{\mu}^{R}$. We then have $\delta\left(\mathcal{M}^{\mu \alpha} F_{\alpha}^{\nu} n_{\mu} n_{\nu}\right)=2 \mathcal{M}_{(0)} \mathcal{B}_{(0)} \delta n_{r}$. Now, using (4.22) we find that $\delta n_{r}=\left[\left(k^{2}-\omega^{2}\right) / 2+m^{2} /\left(2 R_{0}^{2}\right)\right] \epsilon^{2}+\mathcal{O}\left(\epsilon^{3}\right)=$ $\mathcal{O}\left(\epsilon^{2}\right)$. So the magnetic contribution to $\left.\delta \Pi\right|_{\text {bdry }}$ is indeed of order $\epsilon^{2}$ and thus subleading when compared with the rhs of (4.29), that is of order $\epsilon$.
} 


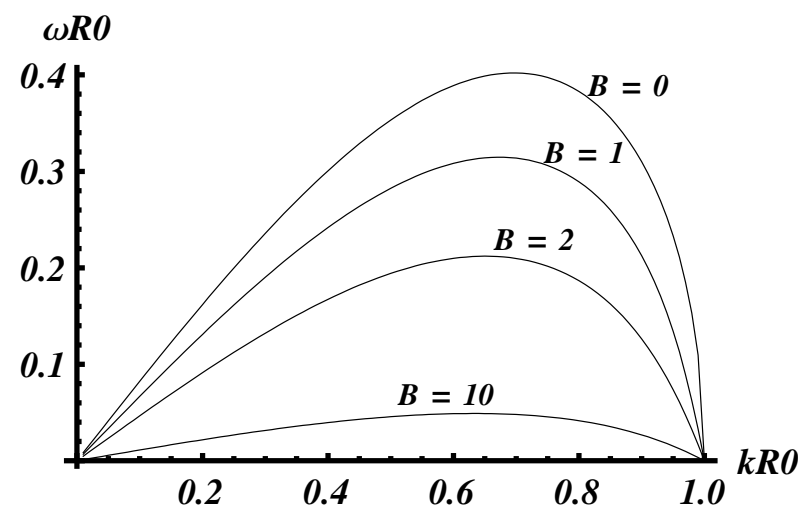

Figure 1: Plot of the dimensionless dispersion relation $\omega(k)$ for the Rayleigh-Plateau instability in a static uniform tube for several values of the axial magnetic field $\mathcal{B}_{(0)}$ (in the plot, $B$ ). The instability strength and decreases as the magnetic field grows. We use (4.32) and the numerical data correspond to take $\frac{\sigma}{\left(\rho_{(0)}+\mathcal{P}_{(0)}\right) R_{0}}=\frac{10^{-6}}{5-2 \mathcal{B}_{(0)}^{2}}$ (see text).

tension potential energy can decrease (to leading order in the perturbation), and thus the condition (4.33) for the instability is independent of the introduction of a background magnetic field ${ }^{18}$. With or without a non-dynamical magnetic field, the Rayleigh-Plateau instability is a long wavelength instability that afflicts the plasma tube when its length is bigger than its transverse radius.

The presence of the non-dynamical magnetic field does however reduce the strength of the instability. Indeed, in the plot of the dispersion relation (4.32), see Fig. 1, we find that an increment in the magnetic field $\mathcal{B}_{(0)}$ weakens the instability. For high values of $\mathcal{B}_{(0)}$ the instability strength becomes considerably weaker. Strictly speaking we cannot however say that it disappears for a critical value of $\mathcal{B}_{(0)}$ since the threshold mode (4.33) is always non-vanishing independently of $\mathcal{B}_{(0)}$.

A word of caution is in order. From the equation of state (4.16) and the equilibrium condition (4.17) one gets for $d=4: \rho_{(0)}+\mathcal{P}_{(0)}=5 a h(\nu, b)-2 \mathcal{B}_{(0)} \mathcal{M}_{(0)}$ (for constant $a$ ). Note that unfortunately we do not know the function $h(\nu, b)$ in five dimensions and this prevents an accurate computation. Depending on its form, the condition $\rho_{(0)}+\mathcal{P}_{(0)}>0$ might require a maximum allowed $\mathcal{B}_{(0)}$. To plot Fig. 1 we took $5 a h(\nu, b) \equiv 1$, i.e., $\rho_{(0)}+\mathcal{P}_{(0)}=5-2 \mathcal{B}_{(0)} \mathcal{M}_{(0)}$, and this indeed implies a critical $\mathcal{B}_{(0)}$. However, we should have in mind that $h$ is not a constant but $h=h(\nu, b)$. There might thus not exist a maximum $\mathcal{B}_{(0)}$.

\subsection{Unstable plasma tubes and their gravitational duals}

The magnetized plasma tubes we have been discussing are dual to magnetic black strings in SS compactified $\mathrm{AdS}_{6}$. The Rayleigh-Plateau (RP) instablity of the plasma tube is dual to the GregoryLaflamme (GL) instability of the black strings. Unfortunately the magnetic black string solutions in SS compactified $\mathrm{AdS}_{6}$ have not been constructed yet, and thus we cannot compare our plasma results with their gravitational duals. Therefore, our study provides solid predictions for the features

\footnotetext{
${ }^{18}$ Later, in the end of next subsection, we will argue that we do no expect this to be true when the approximation $\delta \chi \sim 0$ is relaxed.
} 
and stability of $\mathrm{SS} \mathrm{AdS}_{6}$ magnetic black strings.

There is however growing evidence that justifies the comparison of our plasma results with the properties of magnetically charged black strings in an asymptotically flat background. Let us pause here to review the evidence that seems to permit this extrapolation. In $[2,33]$ the phase diagram of axisymmetric rigidly rotating plasma configurations was found. The set of solutions is given by plasma balls, pinched balls and plasma rings. Quite remarkably, the entropy vs angular momentum (for fixed energy) phase diagram displays remarkable similarities with the phase diagram of asymptotically flat [40,41] and AdS [42] black hole solutions.

More closely connected with our present study, [6] analyzed the RP instability of a neutral plasma tube and the associated phase diagram of non-uniform plasma tubes and localized plasma balls. The plasma instability has properties remarkably similar to the GL instability of a neutral, asymptotically flat black string [7] (see [43,44] for reviews). A brief account of these similarities includes [6]: (i) the dispersion relation of the two instabilities are quite similar and, not less important, both evolve similarly as we add extra dimensions (compare Fig. 5 of [6] with Fig. 3 of [45]); ii) for static configurations, only axisymmetric modes are unstable and there is even a quantitative match for the threshold wavenumber as the number of dimensions gets large; for rotating solutions, non-axisymmetric modes can become unstable in both instabilities; iii) there are similar critical dimensions for the entropically favored solutions; iv) the phase diagram containing the solutions living in a compact dimension, namely (non-)uniform extended configurations and localized configurations is astonishingly similar (compare the $S(J)$ diagram of Fig. 1 of [6] with Fig. 3 of [46]); the known initial time evolution of the GL instability of an asymptotically flat black string is similar to the dynamical evolution of the RP instability (compare Fig. 4 of [47] with Fig. 1 of [48]). General arguments suggest that in the limit of large number of dimensions the fluid description of asymptotically flat black holes should become more and more accurate.

The just mentioned facts motivate us to compare our results for the RP instability of a magnetized plasma tube with the know studies $[45,49,50]$ of the GL instability of magnetically charged asymptotically flat black strings/branes. In $[49,50]$ it is found that a magnetic charge weakens the strength of the GL instability. In view of the above evidence, this is in agreement with our plasma result: a magnetic field also decreases the strength of the RP instability of the plasma tube.

Besides showing that the GL instability gets weaker as magnetic charge is added to the system, $[45,49,50]$ have also been able to show that it actually disappears in the extremal case, when the charge has its maximum allowed value (for some systems it is absent even before the extreme state is reached [50]). This property leads to an interesting new relation. Indeed, a dynamical instability like GL is expected to be accompanied by a local thermodynamic instability. This motivated Gubser and Mitra [51] to propose the conjecture that a black brane/string with a non-compact translational symmetry is classically stable if and only if it is locally thermodynamically stable. A detailed analysis of this conjecture was further done in [52]. In these studies it was indeed found that there is a change in the sign of the specific heat of the black brane precisely for the critical charge where the GL instability disappears. It is thus interesting to discuss a natural version of the Gubser-Mitra conjecture in the uniform plasma tube setup. Unfortunately, we are not able to address this interesting issue. The reason is two-folded. First, to compute the specific heat of the uniform plasma tube we would need to have the relation between the entropy and the energy of 
the system. To get the entropy we would need to integrate the entropy density given by the first relation in (4.15). This requires knowing the precise form of the thermodynamic function $h(\nu, b)$, defined in (4.9), in five dimensions. Unfortunately, this is currently unknown. Second, although the RP instability gets weaker as the magnetic field increases we do not find a finite critical value above which it is not active (see however the discussion at the end of subsection 4.4). We do find that the instability strength reduces substantially as $B$ grows not too large. Although in practice we can say that it disappears for large magnetic field, strictly speaking we are not allowed to say that it is gone since the threshold wavenumber keeps finite (see Fig. 1). The RP modes become sort of marginally stable in the sense that unstable modes are present but with very small strength. So, at most we would be able (if we knew $h(\nu, b)$ ) to check that the specific heat also never becomes positive for the uniform plasma tube as the magnetic field grows. In the uncharged case, $h(\nu, b)$ is a constant, and we found in [6] that the heat capacity is indeed always negative. Adding a small magnetic field should not change this, but we cannot explore the situation for larger fields.

The absence of a critical magnetic field in the RP instability deserves however a much more careful look. As described above this occurs because the threshold wavenumber, where the RP is marginally stable, persists invariant under changes of the magnetic field. This is an odd feature ${ }^{19}$ and it is not hard to convince ourselves that this is a consequence of neglecting the susceptibility perturbations (see discussion associated with (4.19) and footnote 17). Indeed, from footnote 17 we see that when $\delta \chi \neq 0$ there is a contribution proportional to the square of the magnetic field strength in the lhs of (4.29). This contribution persists in the following equations and the threshold wavenumber (4.33) gets then reduced when the magnetic field increases. Unfortunately, when we consider $\delta \chi \neq 0$, the perturbed system is left undetermined even after using the Bianchi identities and the conservation of the polarization current (in footnote 2). We would need to know some microscopic information to express $\delta \chi$ in terms of perturbations of other thermodynamic quantities - a relation that we do not have. For this reason, and because $\delta \chi$ is nevertheless expected to be small (as justified previously), we took $\delta \chi \sim 0$ in our analysis. This approximation nevertheless allowed to find that a magnetic field decreases the RP instability strength, and we have a good control on the effects that a non-vanishing susceptibility perturbation introduces in the system: the instability strength still decreases when $B$ grows and, on the top of this, the critical wavenumber also decreases.

Usually an unstable mode signals a bifurcation to a new branch of configurations in the phase diagram of solutions. Without magnetic fields this is indeed the case and the RP unstable mode of the uniform plasma tube leads to a branch of non-uniform plasma tubes that joins a third branch of plasma balls localized on the compact tube direction $[6,54]$. Although not studied here, again because we do not know the function $h(\nu, b)$, we naturally expect that similar new branches of solutions, representing magnetic non-uniform tubes and magnetic localized plasma balls, exist also in the phase diagram of magnetized plasma configurations.

To end this section, we address the effects that viscosity introduces in the Rayleigh-Plateau instability. These were already discussed in more detail in [6], so we will be brief in our comments.

\footnotetext{
${ }^{19}$ Indeed, if we consider the standard RP instability on a charged fluid tube coupled to a dynamical magnetic field, we find that both the instability strength and the critical wavenumber decrease when the magnetic field increases [53].
} 
Generically, at least classicaly, the viscosity increases the wavelength of the most unstable mode, and weakens the strength of the instability [53]. It has a subleading effect on the activation of the instability (however, the instability can get considerably weaker if the viscosity is very high), but plays an important role at later stages in the time evolution of the instability, namely in the pinch-off phase of the plasma tube [48]. Typically, the lower the viscosity is, the higher is the number of plasma balls formed. Your fluid is non-conformal so we have bulk and shear viscosity and thermal dissipation. Dissipation increases considerably the technical challenge of solving (even numerically) the MHD perturbed equations: we get a coupled system of differential equations with a fourth order derivative term.

\subsection{Regime of validity}

To conclude this section, the regime of validity of our results should be kept in mind.

First of all, the fluid description of the deconfined plasma must be accurate, and hence the thermodynamic quantities must vary slowly over the mean free path $\ell_{\mathrm{mfp}}$ of the constituent particles, which is of the order of the mass gap of the theory, or equivalently of the order of the deconfinement temperature $T_{c} \sim \frac{\rho_{0}}{\sigma}$. That is, all length scales $\lambda$ in the fluid must be

$$
\lambda \gg T_{c}^{-1} \sim \frac{\sigma}{\rho_{0}} .
$$

This condition imposes restrictions on the validity of the Rayleigh-Plateau instability analysis: the $\mathrm{RP}$ unstable frequencies and wavenumbers must satisfy

$$
\left\{\omega R_{0}, k R_{0}\right\} \gg \frac{\sigma}{\rho_{0} R_{0}} .
$$

Since the most unstable mode dominates the instability we must guarantee that this condition is verified in the vicinity of the maximum of the dispersion relation. We find that for $\frac{\sigma}{\rho_{0} R_{0}} \lesssim 10^{-4}$ this relation is satisfied, and things get better as $\frac{\sigma}{\rho_{0} R_{0}}$ becomes smaller. In particular this is true in the dispersion relation plot of Fig. 1.

Second, the interface between the confined and deconfined phases, i.e., the fluid surface, has a finite thickness of the order $1 / T_{c}[32]$, and therefore the delta-like surface approximation we used is valid provided that the curvature of the surface is small with respect to the scale $1 / T_{c}$. So, for plasma tubes the analysis is good when the boundary radius $R_{o}$ is everywhere large when compared with $T_{c}^{-1}$,

$$
\frac{\sigma}{\rho_{0} R_{0}} \ll 1
$$

which is perfectly compatible with (4.35).

Finally, we neglected the dependence of the surface tension on the temperature and other thermodynamic quantities of the fluid. For consistency we must then demand that on the boundary between the confined and deconfined phases the temperature of the plasma must remain everywhere close to the critical temperature $T_{c}$, i.e., $\mathcal{T} / T_{c} \sim 1$ at the tube boundary.

As discussed already in great detail in subsection 4.5, we also neglected the dependence of the magnetic susceptibility on the temperature and on other thermodynamic quantities. In the previous section we already identified the effects of taking this assumption and of relaxing it. 
Once these conditions are fulfilled, the plasma approximation holds and can be trusted to study the properties of black holes. The moral of this analysis is that there is a broad window of parameters for which the analysis of the Rayleigh-Plateau instability is well within the required validity regime.

\section{Acknowledgements}

We thank Roberto Emparan for useful discussions. MC and OJCD are grateful to the organizers of the workshop "Quantum Black Holes, Braneworlds and Holography", Valencia, May 2008; OJCD and DK thank CERN for hospitality during the programme "Black Holes: A Landscape of Theoretical Physics Problems", August-October 2008; OJCD thanks the Niels Bohr Institute for hospitality and the organizers of the workshop "Mathematical Aspects of General Relativity", Copenhagen, April 2008, where part of this work was done. MC was supported in part by DURSI 2005 SGR 00082, MEC FPA 2004-04582-C02 and FPA-2007-66665-C02, the European Community FP6 program MRTN-CT-2004-005104, the FWO - Vlaanderen, project G.0235.05 and in part by the Federal Office for Scientic, Technical and Cultural Affairs through the Interuniversity Attraction Poles Programme Belgian Science Policy P6/11-P. OJCD acknowledges financial support provided by the European Community through the Intra-European Marie Curie contract MEIF-CT-2006038924. This work was partially funded by FCT through project PTDC/FIS/64175/2006. DK was supported in part by INFN, MIUR-PRIN contract 20075ATT78, and by the European Community FP6 program MRTN-CT-2004-005104.

\section{References}

[1] O. Aharony, S. S. Gubser, J. M. Maldacena, H. Ooguri and Y. Oz, "Large $N$ field theories, string theory and gravity," Phys. Rept. 323 (2000) 183 [arXiv:hep-th/9905111].

[2] S. Lahiri and S. Minwalla, "Plasmarings as dual black rings," arXiv:0705.3404 [hep-th].

[3] J. Evslin and C. Krishnan, "Metastable black saturns," JHEP 0809 (2008) 003 [arXiv:0804.4575 [hep-th]].

[4] S. Bhattacharyya, V. E. Hubeny, S. Minwalla and M. Rangamani, "Nonlinear fluid dynamics from gravity," JHEP 0802 (2008) 045 [arXiv:0712.2456 [hep-th]].

[5] M. Van Raamsdonk, "Black hole dynamics from atmospheric science," JHEP 0805 (2008) 106 [arXiv:0802.3224 [hep-th]].

[6] M. M. Caldarelli, O. J. C. Dias, R. Emparan and D. Klemm, "Black holes as lumps of fluid," arXiv:0811.2381 [hep-th].

[7] R. Gregory and R. Laflamme, "Black strings and p-branes are unstable," Phys. Rev. Lett. 70 (1993) 2837 [arXiv:hep-th/9301052]. 
[8] S. A. Hartnoll, C. P. Herzog and G. T. Horowitz, "Building an AdS/CFT superconductor," arXiv:0803.3295 [hep-th].

[9] S. S. Gubser, "Breaking an Abelian gauge symmetry near a black hole horizon," arXiv:0801.2977 [hep-th].

[10] S. A. Hartnoll and P. Kovtun, "Hall conductivity from dyonic black holes," Phys. Rev. D 76 (2007) 066001 [arXiv:0704.1160 [hep-th]].

[11] S. A. Hartnoll, P. K. Kovtun, M. Müller and S. Sachdev, "Theory of the Nernst effect near quantum phase transitions in condensed matter, and in dyonic black holes," Phys. Rev. B 76 (2007) 144502 [arXiv:0706.3215 [cond-mat.str-el]].

[12] G. Compère and D. Marolf, "Setting the boundary free in AdS/CFT," Class. Quant. Grav. 25 (2008) 195014 [arXiv:0805.1902 [hep-th]].

[13] S. Bhattacharyya, S. Lahiri, R. Loganayagam and S. Minwalla, "Large rotating AdS black holes from fluid mechanics," arXiv:0708.1770 [hep-th].

[14] J. Hansen and P. Kraus, "Nonlinear magnetohydrodynamics from gravity," arXiv:0811.3468 [hep-th].

[15] N. Andersson and G. L. Comer, "Relativistic fluid dynamics: Physics for many different scales," Living Rev. Rel. 10 (2005) 1 [arXiv:gr-qc/0605010].

[16] A. Ishibashi and R. M. Wald, "Dynamics in non-globally hyperbolic static spacetimes. III: anti-de Sitter spacetime," Class. Quant. Grav. 21 (2004) 2981 [arXiv:hep-th/0402184].

[17] D. Marolf and S. F. Ross, "Boundary conditions and new dualities: Vector fields in AdS/CFT," JHEP 0611 (2006) 085 [arXiv:hep-th/0606113].

[18] V. Balasubramanian and P. Kraus, "A stress tensor for anti-de Sitter gravity," Commun. Math. Phys. 208 (1999) 413 [arXiv:hep-th/9902121].

[19] M. Bianchi, D. Z. Freedman and K. Skenderis, "Holographic Renormalization," Nucl. Phys. B 631 (2002) 159 [arXiv:hep-th/0112119].

[20] A. M. Awad and C. V. Johnson, "Holographic stress tensors for Kerr-AdS black holes," Phys. Rev. D 61 (2000) 084025 [arXiv:hep-th/9910040].

[21] D. T. Son and A. O. Starinets, "Viscosity, Black Holes, and Quantum Field Theory," Ann. Rev. Nucl. Part. Sci. 57 (2007) 95 [arXiv:0704.0240 [hep-th]].

[22] M. M. Caldarelli, G. Cognola and D. Klemm, "Thermodynamics of Kerr-Newman-AdS black holes and conformal field theories," Class. Quant. Grav. 17 (2000) 399 [arXiv:hep-th/9908022].

[23] S. W. Hawking, C. J. Hunter and M. Taylor, "Rotation and the AdS/CFT correspondence," Phys. Rev. D 59 (1999) 064005 [arXiv:hep-th/9811056]. 
[24] S. Bhattacharyya, R. Loganayagam, S. Minwalla, S. Nampuri, S. P. Trivedi and S. R. Wadia, "Forced Fluid Dynamics from Gravity," arXiv:0806.0006 [hep-th].

[25] A. H. Chamseddine and W. A. Sabra, "Magnetic strings in five-dimensional gauged supergravity theories," Phys. Lett. B 477 (2000) 329 [arXiv:hep-th/9911195].

[26] D. Klemm and W. A. Sabra, "Supersymmetry of black strings in $D=5$ gauged supergravities," Phys. Rev. D 62 (2000) 024003 [arXiv:hep-th/0001131].

[27] K. Copsey and G. T. Horowitz, "Gravity dual of gauge theory on $S^{2} \times S^{1} \times \mathbb{R}$," JHEP 0606 (2006) 021 [arXiv:hep-th/0602003].

[28] R. B. Mann, E. Radu and C. Stelea, "Black string solutions with negative cosmological constant," JHEP 0609 (2006) 073 [arXiv:hep-th/0604205].

[29] Y. Brihaye, E. Radu and C. Stelea, "Black strings with negative cosmological constant: Inclusion of electric charge and rotation," Class. Quant. Grav. 24 (2007) 4839 [arXiv:hepth/0703046].

[30] A. Bernamonti, M. M. Caldarelli, D. Klemm, R. Olea, C. Sieg and E. Zorzan, "Black strings in $\mathrm{AdS}_{5}, "$ JHEP 0801 (2008) 061 [arXiv:0708.2402 [hep-th]].

[31] T. Delsate, "New stable phase of non uniform black strings in $\mathrm{AdS}_{d}$," arXiv:0808.2752 [hep-th].

[32] O. Aharony, S. Minwalla and T. Wiseman, "Plasma-balls in large N gauge theories and localized black holes," Class. Quant. Grav. 23 (2006) 2171 [arXiv:hep-th/0507219].

[33] S. Bhardwaj and J. Bhattacharya, "Thermodynamics of Plasmaballs and Plasmarings in 3+1 Dimensions," arXiv:0806.1897 [hep-th].

[34] V. Cardoso and O. J. C. Dias, "Gregory-Laflamme and Rayleigh-Plateau instabilities," Phys. Rev. Lett. 96 (2006) 181601 [arXiv:hep-th/0602017].

[35] V. Cardoso and L. Gualtieri, "Equilibrium configurations of fluids and their stability in higher dimensions," Class. Quant. Grav. 23 (2006) 7151 [arXiv:hep-th/0610004].

[36] V. Cardoso, O. J. C. Dias and L. Gualtieri, "The return of the membrane paradigm? Black holes and strings in the water tap," Int. J. Mod. Phys. D 17 (2008) 505 [arXiv:0705.2777 [hep-th]].

[37] O. J. C. Dias, T. Harmark, R. C. Myers and N. A. Obers, "Multi-black hole configurations on the cylinder," Phys. Rev. D 76, 104025 (2007) [arXiv:0706.3645 [hep-th]].

[38] U. Miyamoto and K. i. Maeda, "Liquid bridges and black strings in higher dimensions," Phys. Lett. B 664 (2008) 103 [arXiv:0803.3037 [hep-th]].

[39] U. Miyamoto, "Curvature driven diffusion, Rayleigh-Plateau, and Gregory-Laflamme," Phys. Rev. D 78 (2008) 026001 [arXiv:0804.1723 [hep-th]]. 
[40] R. Emparan and H. S. Reall, "A rotating black ring in five dimensions," Phys. Rev. Lett. 88 (2002) 101101 [arXiv:hep-th/0110260].

[41] R. Emparan, T. Harmark, V. Niarchos, N. A. Obers and M. J. Rodriguez, "The Phase Structure of Higher-Dimensional Black Rings and Black Holes," JHEP 0710 (2007) 110 [arXiv:0708.2181 [hep-th]].

[42] M. M. Caldarelli, R. Emparan and M. J. Rodriguez, "Black Rings in (Anti)-deSitter space," arXiv:0806.1954 [hep-th].

[43] B. Kol, "The phase transition between caged black holes and black strings: A review," Phys. Rept. 422 (2006) 119 [arXiv:hep-th/0411240].

[44] T. Harmark, V. Niarchos and N. A. Obers, "Instabilities of black strings and branes," Class. Quant. Grav. 24 (2007) R1 [arXiv:hep-th/0701022].

[45] R. Gregory and R. Laflamme, "The Instability of charged black strings and p-branes," Nucl. Phys. B 428 (1994) 399 [arXiv:hep-th/9404071].

[46] T. Harmark and N. A. Obers, "Phases of Kaluza-Klein black holes: A brief review," arXiv:hepth/0503020.

[47] M. W. Choptuik, L. Lehner, I. Olabarrieta, R. Petryk, F. Pretorius and H. Villegas, "Towards the final fate of an unstable black string," Phys. Rev. D 68 (2003) 044001 [arXiv:grqc/0304085].

[48] M. Tjahjadi, H. A. Stone and J. M. Ottino, "Satellite and subsatellite formation in cappilary breakup," J. Fluid Mech. 243 (1992) 297.

[49] R. Gregory and R. Laflamme, "Evidence For Stability Of Extremal Black P-Branes," Phys. Rev. D 51 (1995) 305 [arXiv:hep-th/9410050].

[50] T. Hirayama, G. w. Kang and Y. o. Lee, "Classical stability of charged black branes and the Gubser-Mitra conjecture," Phys. Rev. D 67 (2003) 024007 [arXiv:hep-th/0209181].

[51] S. S. Gubser and I. Mitra, "Instability of charged black holes in anti-de Sitter space," arXiv:hepth/0009126.

[52] H. S. Reall, "Classical and thermodynamic stability of black branes," Phys. Rev. D 64 (2001) 044005 [arXiv:hep-th/0104071].

[53] S. Chandrasekhar, "Hydrodynamic and hydromagnetic stability" (Dover Publications, New York, 1981).

[54] K. i. Maeda and U. Miyamoto, "Black hole-black string phase transitions from hydrodynamics," arXiv:0811.2305 [hep-th]. 